\title{
Prevalence, incidence and bothersomeness of urinary incontinence between 6 weeks and 1 year post-partum: a systematic review and meta-analysis
}

\author{
Heidi F. A. Moossdorff-Steinhauser ${ }^{1} \cdot$ Bary C. M. Berghmans $^{2} \cdot$ Marc E. A. Spaanderman $^{3} \cdot$ Esther M. J. Bols $^{1}$
}

Received: 27 January 2021 / Accepted: 20 May 2021 / Published online: 17 June 2021

(C) The Author(s) 2021

\begin{abstract}
Introduction and hypothesis Urinary incontinence (UI) is a common complaint for post-partum women. Reported prevalence and incidence figures show a large range due to varying study methodology. The crude prevalence of post-partum UI may differ when accounting for bother. Precise prevalence and incidence figures on (bothersome) UI are of relevance for health care providers, research planning, and policy makers. Therefore, we conducted a systematic review and meta-analysis to investigate the prevalence and incidence of UI in post-partum women in the Western world for relevant subgroups and assessed experienced bother in relation to UI.

Methods Observational studies, published between January 1998 and March 2020 and reporting on prevalence and incidence between 6 weeks and 1 year post-partum, were included, regardless of type of UI or setting. We used a random effects model with subgroup analyses for post-partum period, parity and subtype of UI.

Results The mean (weighted) prevalence based on 24 included studies, containing a total of 35.064 women, was $31.0 \%$. After an initial drop in prevalence at 3 months post-partum, prevalence rises up to nearly the same level as in the third trimester of pregnancy at 1 year post-partum (32\%). Stress UI (54\%) is the most prevalent type. UI prevalence is equal among primi- and multiparous women. Experienced bother of UI is heterogeneously assessed and reported to be mild to moderate.

Conclusions Post-partum UI is highly prevalent in women in the Western world. After an initial drop it rises again at 1 year postpartum. Experienced bother is mild to moderate.
\end{abstract}

Keywords Bother $\cdot$ Incidence $\cdot$ Post-partum $\cdot$ Prevalence $\cdot$ Systematic review $\cdot$ Urinary incontinence

\section{Introduction}

Urinary incontinence (UI) is the complaint of involuntary loss of urine [1]. The main subtypes of UI are stress (S) UI, urgency (U) UI and mixed (M) UI. SUI is leaking urine when coughing or sneezing [1]. SUI is more common in younger women [2].

Heidi F. A. Moossdorff-Steinhauser

heidi.moossdorff@maastrichtuniversity.nl

1 Faculty of Health, Medicine and Life Sciences, Department of Epidemiology, Care and Public Health Research Institute (CAPHRI), Maastricht University, P.O. Box 616, 6200, MD Maastricht, The Netherlands

2 Pelvic care Center Maastricht, CAPHRI, Maastricht University Medical Centre (MUMC+), Maastricht, The Netherlands

3 Department of Obstetrics and Gynaecology, MUMC+, Maastricht, The Netherlands
Pregnancy and vaginal delivery are well-documented risk factors for developing UI [3-5]. Seventy-three percent of women with UI 3 months post-partum still report UI at 6 years postpartum [6]. In general, UI prevalence and incidence rise with ageing [7]. Women often experience UI as embarrassing and humiliating, resulting in loss in quality of life [8]. UI also causes considerable socio-economic costs $[9,10]$.

The prevalence and incidence of UI in the post-partum period are widely studied. However, these prevalence and/or incidence figures vary greatly throughout published reports, depending on local setting, case definitions applied, recruited population (period post-partum and parity) and study methodology [11, 12]. A systematic review on the prevalence of post-partum UI and the relation to the mode of delivery was published in 2010 [13]. At that time, studies hardly reported on bother. In 2017, the International Consultation on Incontinence (ICI) recommended that prevalence numbers should be accompanied by the experienced bother [14], as there are indications that the prevalence of 
bothersome UI is lower than the crude UI prevalence [14]. As women with bothersome UI tend to seek more help [15], health professionals, policy makers and researchers need reliable prevalence numbers to specify the health problem UI causes and to help set priorities and assist in planning the management of UI.

Therefore, the primary aim of this systematic review and meta-analysis was to examine the pooled overall prevalence and incidence of UI between 6 weeks and 1 year post-partum in the general population of the Western world, specified for relevant subcategories (period post-partum, parity, type of UI, frequency and amount). A secondary aim was to provide an overview of the assessment methods and outcomes for bother in relation to UI as used in included studies.

\section{Methods}

The MOOSE statement for reporting systematic reviews and meta-analyses was followed [16]. The research protocol was published in the PROSPERO database (registration number CRD42018111991).

\section{Search strategy}

We performed a systematic review and meta-analysis of observational studies (cross-sectional and cohort studies) reporting on the prevalence and incidence [17] of UI after delivery and experienced bother. We searched the electronic databases of PubMed, EMBASE and CINAHL. All included articles were reference checked. Titles and/or abstracts of studies retrieved using the search strategy and those from additional sources were screened independently by two reviewers. Full texts of potentially eligible studies were retrieved and independently assessed for eligibility by two review team members. Any disagreement on eligibility was resolved through discussion with a third reviewer.

We used the following search terms to search all databases: postpartum, post-partum, post partum, peripartum, peri-partum, peri partum, primiparous, multiparous, multigrav*, multipar*, urinary incontinence, urine loss, leaking urine, incontinence, prevalence, incidence, epidemiology, frequency, bothersomeness, bother*, quality of life and hindrance. In the Appendix the complete search strategy for PubMed is provided. This search string was adapted for use in the other databases.

\section{Eligibility criteria}

Observational studies published between January 1, 1998, and March 1, 2020, in Dutch, English, German and French were included. All studies examining prevalence and/or incidence of UI from 6 weeks to 12 months post-partum among adult primi- and multiparous women in the Western world, regardless of type of UI and setting, were of interest. Six weeks post- partum was chosen to ensure a large proportion of the sample had recovered physiologically from the delivery. Outcomes of interest were prevalence and/or incidence of (bothersome) UI. Exclusion criteria were: articles not available in full or not reporting an overall UI prevalence and/or incidence of any frequency, studies examining only twin pregnancies and studies originating from non-Western countries. The latter criteria were chosen for the purpose of homogeneity in population characteristics. When articles did not report a prevalence figure or response rate, an attempt was made for estimation from the information provided. Throughout this article we use the term bother (in relation to UI) as an umbrella term for related constructs [impact on daily life or quality of life (QOL)].

\section{Study selection}

Titles and/or abstracts of studies retrieved using the search strategy and those from additional sources were screened independently by two reviewers (HM and EB) to identify studies that potentially meet the inclusion criteria outlined above. The full texts of these potentially eligible studies were retrieved and independently assessed for eligibility by these two reviewers. Any disagreement on eligibility was resolved through discussion with a third reviewer (BB). All the included articles were reference checked.

\section{Data extraction and risk of bias}

Information on each study was extracted in a standardized data extraction form, based on the Cochrane Public Health Data Extraction and Assessment template [18]. To assess the risk of bias, the Joanna Briggs critical appraisal tool for studies reporting prevalence data was used $[19,20]$. The checklist consists of nine questions, with the response options yes, no, unclear or not applicable. Overall risk of study bias was rated as low (defined as $8-9$ criteria answered as 'yes'), moderate (4-7 criteria answered as 'yes') or high risk ( $\leq 3$ criteria answered as 'yes'). The response option not applicable (occasionally scored in criteria 5) was considered to be a 'yes'. Two reviewers (HM, EB) extracted data independently. Inconsistencies were identified and resolved through discussion including a third author (BB) if necessary.

Characteristics regarding measurement instruments for bother were extracted in a separate standardized extraction form. The form contains items such as measurement instrument, related construct and measurement results.

\section{Summary measures, statistical analyses and heterogeneity}

We used a random effects model to pool the inverse variance (IV) weighted prevalence of UI in individuals to avoid undue influence on the summary estimate from smaller and less 
precise studies or studies with a very small prevalence. Pooled prevalence and incidence values were reported with $95 \%$ confidence intervals (CI). The degree of heterogeneity was determined by the $\mathrm{I}^{2}$ statistic, with $\mathrm{I}^{2}>75 \%$ labelled as high heterogeneity [21].

Prevalence was studied by subgroup [post-partum period (6 weeks, 3, 6, 9 and 12 months), type and frequency of UI, and parity (primi- and multiparous)] as this might explain why studies show varying prevalence figures. Studies reporting on a post-partum period other than the five established periods are classified in the closest post-partum period. Moreover, studies reporting a period prevalence (e.g. 9-12 months post-partum) are classified in the upper range of the period prevalence (i.e. 12 months), as most women will most likely report on their current status, which is less prone to recall bias. Incidence is reported in two periods: from delivery up to and including 3 months post-partum and from 3 to 12 months post-partum and for primi- and multiparous women. STATA Statistical Software, release 15, was used for analysis.

To determine the overall experienced bother in relation to UI across included studies, the measurement results of the different measurement instruments for bother were converted, where possible, to a (standardized) 0 to 100 scale, with 0 indicating no bother and 100 indicating extremely bothered. We classified 1 to 20 as no to mild bother, 20 to 40 as mild to moderate bother, 40 to 60 as moderate to severe, 60 to 80 as severe to very severe and 80 to 100 as extremely severe bother. We used the following conversion method for the ICIQ-UI SF (range $0-21$ ): converted score $=$ observed original score $*$ 4.76 (the value 4.76 is derived from 100 (upper limit converted score)/21 (upper limit original score). Likewise, question 3 from the ICIQ-UI SF (range $0-10$ ) is calculated as follows: converted score $=$ observed original score $* 10$.

\section{Results}

\section{Study selection}

Among the 1063 papers initially identified, 31 met the eligibility criteria (Fig. 1), resulting in a total of 38,209 participants. All included studies were observational (20 cohort studies $[5,11,12,22-38]$ and 11 cross-sectional studies [39-49]) and published between 1998 and March 1, 2020. Studies were excluded based on inadequate study design, study population, non-Western countries, outcome, follow-up or language. Twenty-nine studies reported on prevalence and/or incidence figures and two studies only reported on incidence figures.

\section{Risk of bias}

The risk of bias for each study is shown in Table 1. High, moderate and low risk of bias was considered to be present in 1,26 and 4 studies respectively. Risk-of-bias items with the lowest ratings were 8 and 9, and risk-of-bias items with the highest ratings were 1 and 3 .

\section{Study characteristics}

The studies originated from Europe $(n=17)$, North America $(n=8)$ and Australia $(n=5)$. One study was mixed (Europe/ Australia). The majority of women were included from a (tertiary) hospital $(n=26)$. The remaining studies included women from the community, primary health care service or health care insurance service. Nineteen studies only reported on primiparous women. Twelve studies used validated questionnaires to determine the presence of UI, and 19 studies used self-constructed, non-validated questionnaires. Table 1 summarizes the study characteristics of included studies.

Nine studies reported on (measurement instruments for) bother. Table 2 provides an overview of the measurement instruments as used in the included studies, with the original and the converted (0-100 scale) measurement results. Five different measurement instruments for bother were used, of which the ICIQ-UI SF was most frequently used [27, 29, 37, 39]. One study only reported the results of the ICIQ-UI SF as categories [50], and two studies did not report total scores [24, 45]. One measurement instrument was self-constructed and non-validated [30].

\section{Synthesis of results}

\section{Overall prevalence}

Twenty-four out of 31 studies contributed to the calculation of the overall prevalence of post-partum UI, involving a total of 35,064 women. The weighted mean of UI prevalence among post-partum women (6 weeks to 12 months) was $31.0 \%$ (CI $95 \% 26.0-36.0 \%$; $\mathrm{I}^{2}: 99.0 \%$ ), regardless of parity or type of UI (Fig. 2). The lowest prevalence of UI found in the included studies was $10 \%$ [51] and the highest prevalence 63\% [52]. Prevalence figures for studies with low $(n=3)$, moderate $(n=$ 20 studies) and high risk of bias $(n=1)$ were $28 \%(95 \%$ CI $17.0-39.0), 29 \%$ (95\% CI 24.0-35.0) and 63\%, respectively (Table 1).

\section{Subcategories post-partum period, type of UI and parity}

Figure 3 summarizes the mean UI prevalence at 6 weeks, 3, 6, 9, and 12 months post-partum. From an initial drop in (weighted) prevalence between 6 weeks $[24.0 \%, 95 \% \mathrm{CI}$ : $17.0-32.0 \%(1349 / 5137)]$ and 3 months post-partum [21.0\%, 95\% CI: $17.0-25.0 \%(3677 / 17,165)]$, prevalence numbers gradually rise to $32.0 \%$ at 12 months post-partum (95\% CI: $23.0-41.0 \%$ (2997/9220)). The prevalence of UI post-partum is equal among primi- and multiparous women, $31 \%[11,12$, 


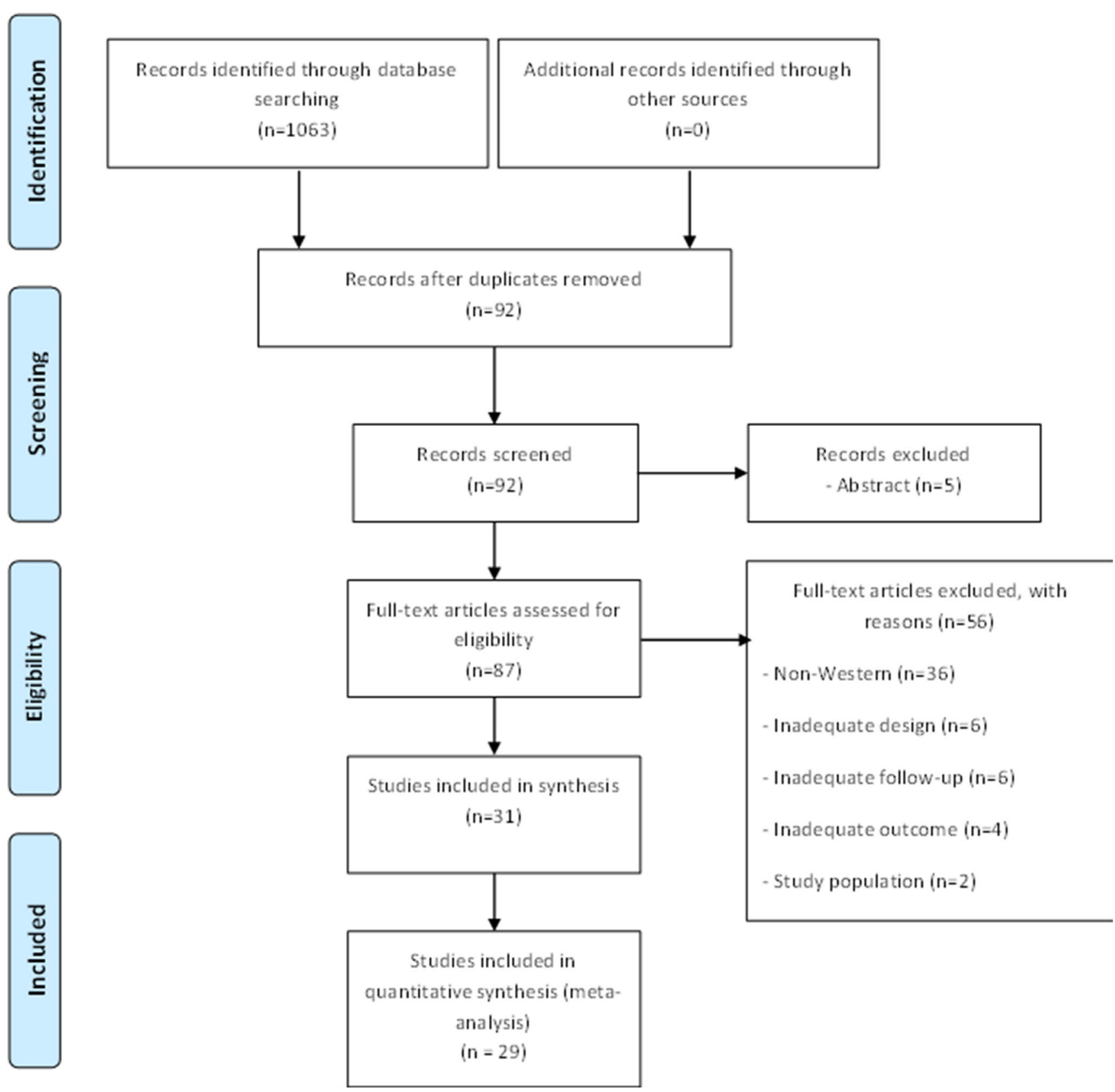

Fig. 1 Study flow diagram

$22-24,26,27,36,37,42,44,48,49,53]$ and $30 \%[30,33-35$, $40,43,45-47,54]$, respectively.

Based on nine studies, SUI accounts for 54\% UUI and MUI for $26 \%$ and $16 \%$ of cases respectively, whereas $4 \%$ was unexplained UI [23, 24, 27, 34, 36, 37, 44, 47, 48].

\section{Subcategories frequency and amount of UI}

Seven out of 31 studies reported on frequency of UI. The most used frequency categories $(n=3)$ were: less than once a week, less than daily, more than or equal once per week and more than or equal daily leakage. A frequency of less than once a week was most frequently reported (50\%$66.3 \%)$ [ $[23,46,55]$. Two studies reported frequency of UI as: less than once per month, a few times a month, a few times a week, every day and/or night $[34,50]$. One study reported: occasionally, once per week, several times per week and daily [32] and one study reported the ICIQ-UI SF question on frequency [27].

Four studies reported on the amount of urine loss $[5,27,34$, 50]. One study used the ICIQ-UI SF to assess this parameter (none, small, moderate, large amount) [27]. One study reported the ICIQ-UI SF item 'amount' separately, showing that the majority of UI patients lose a small amount (85.3\%) [27]. 


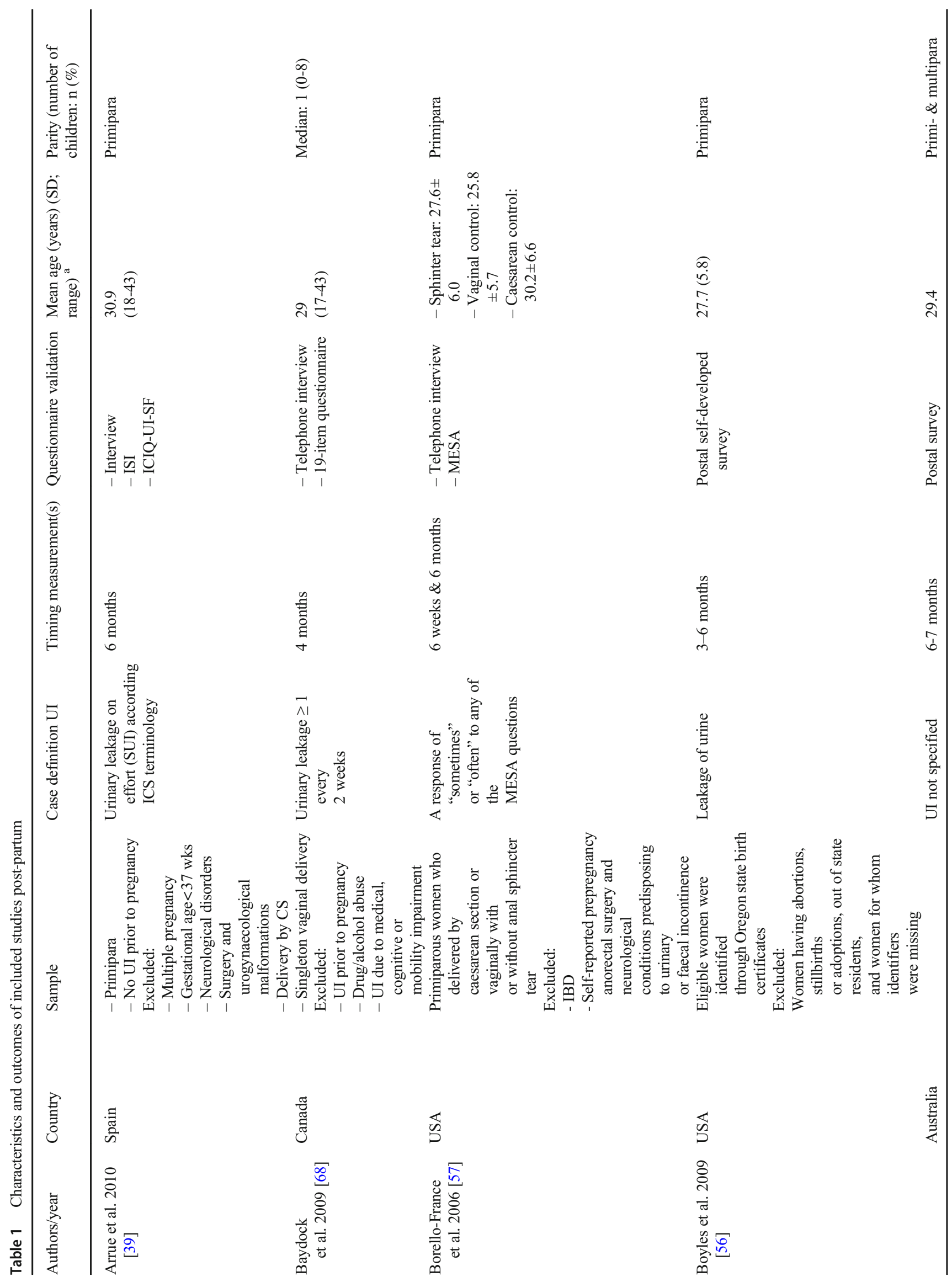




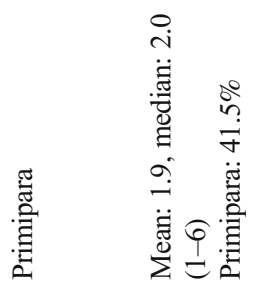

苛

劳

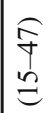

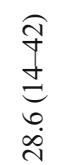

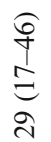

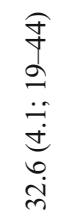
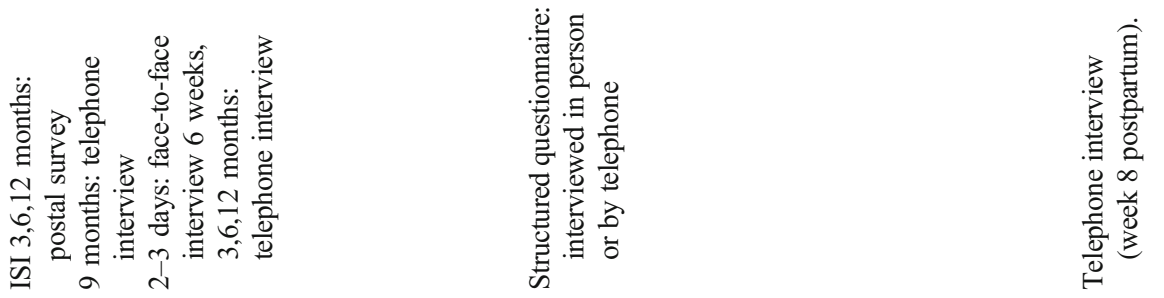

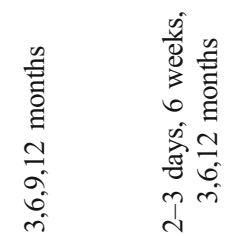

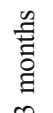

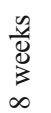

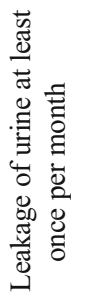

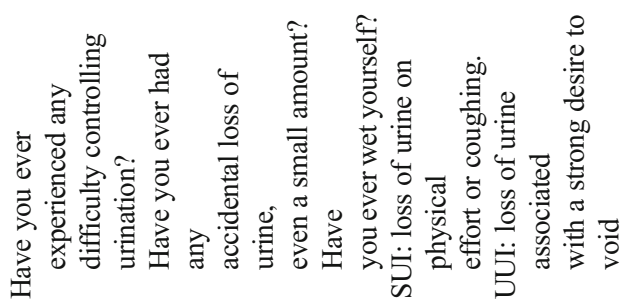

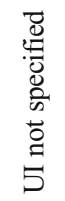

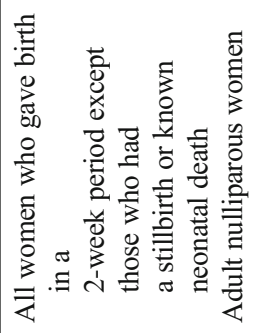
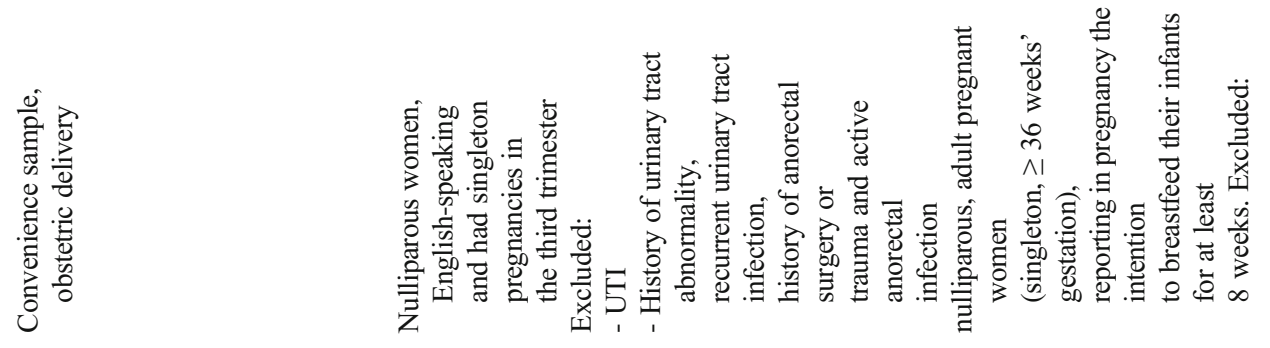

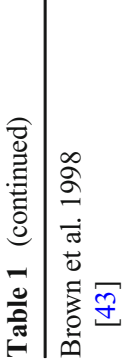
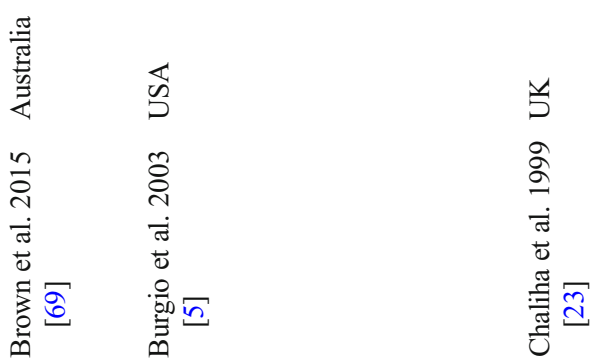

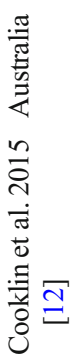




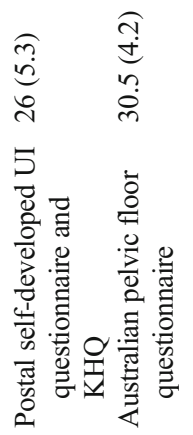

营

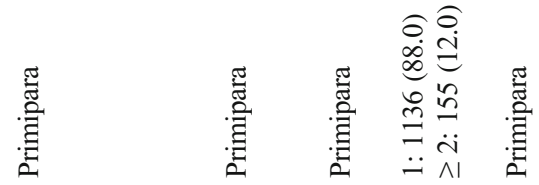

营 芯

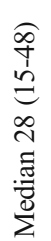

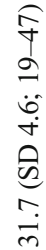

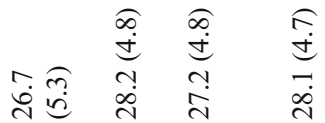
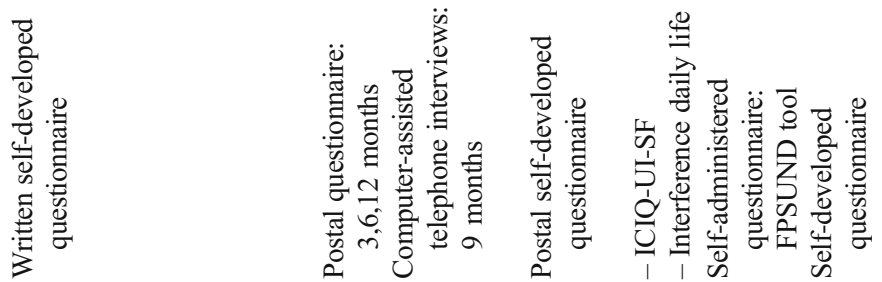

ก

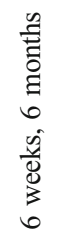

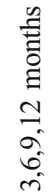
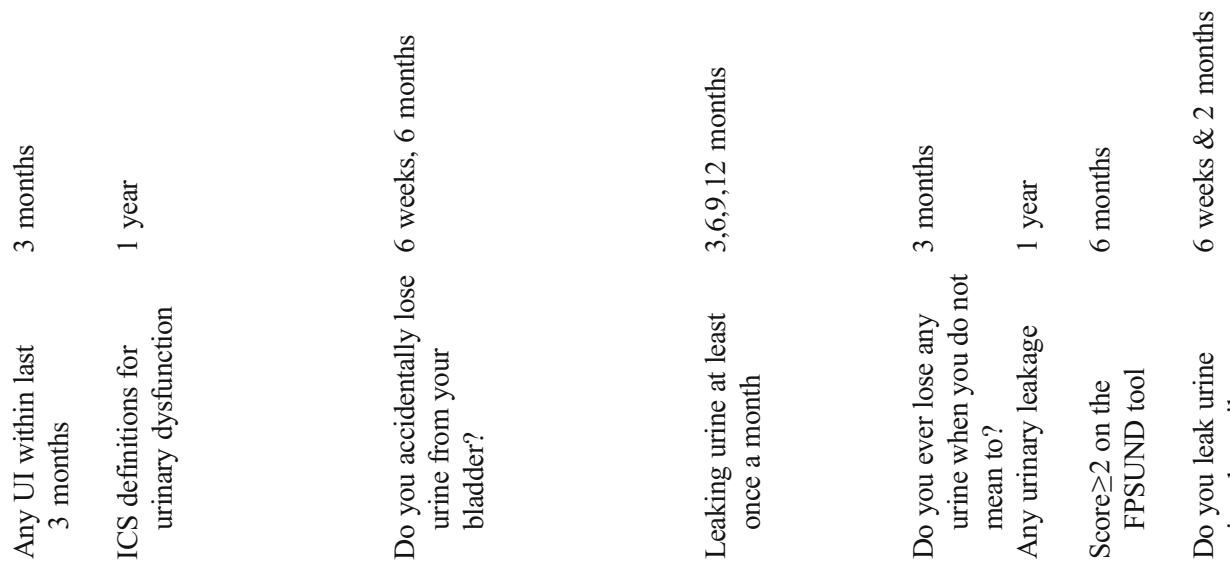

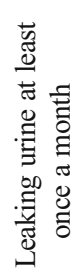
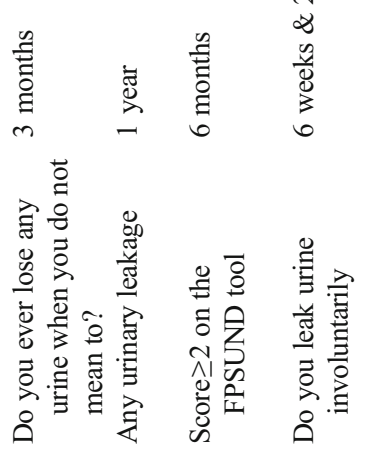

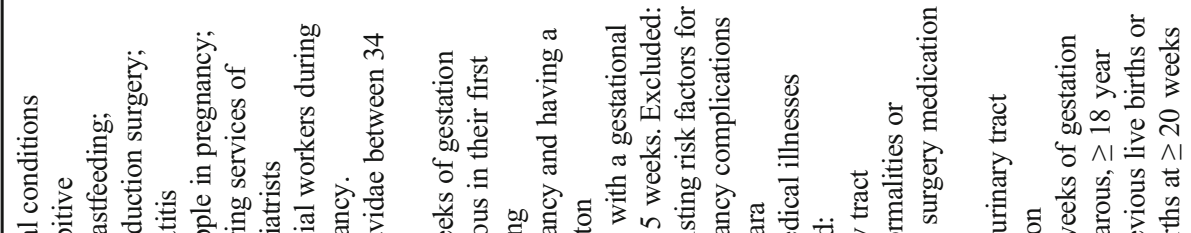

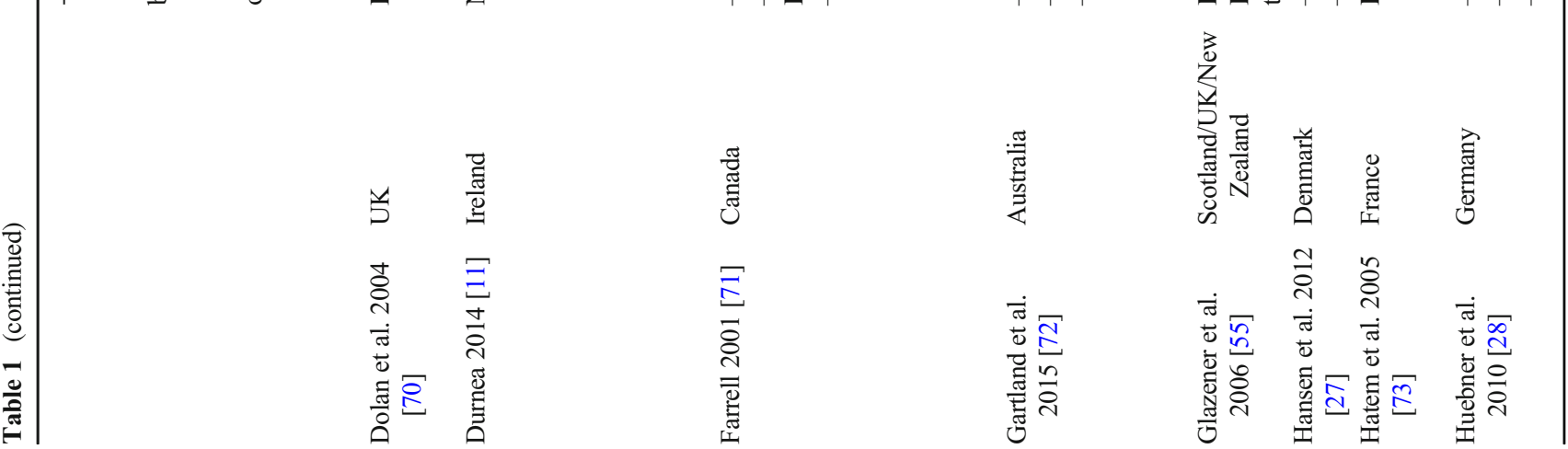

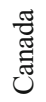

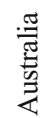

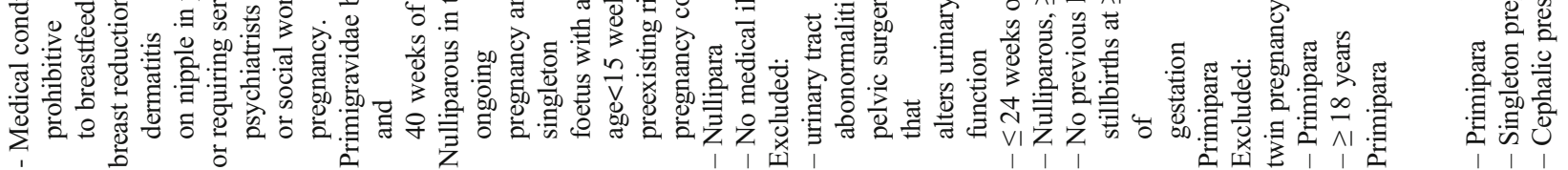



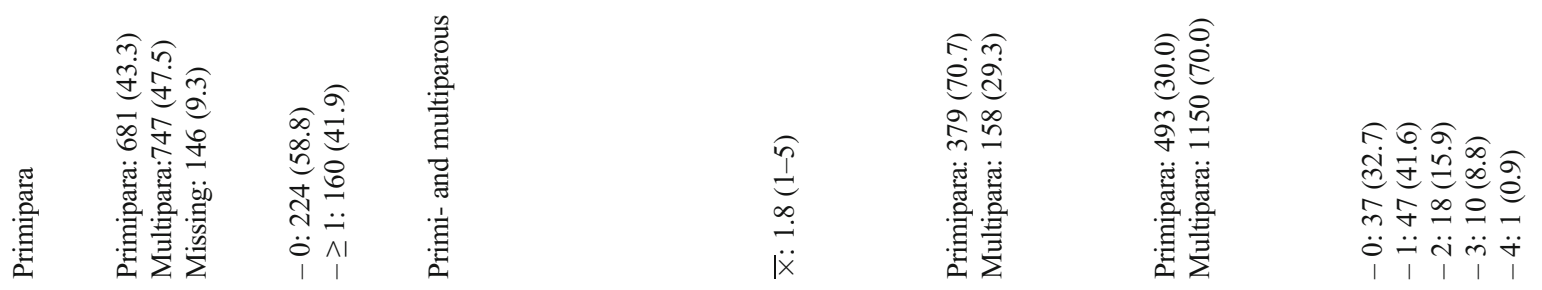

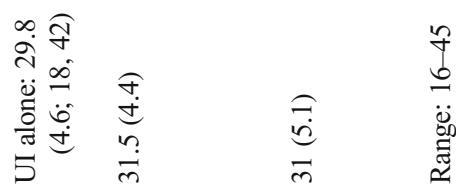

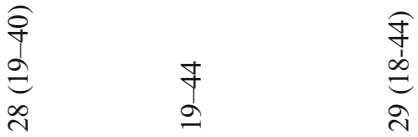

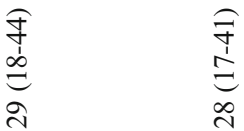

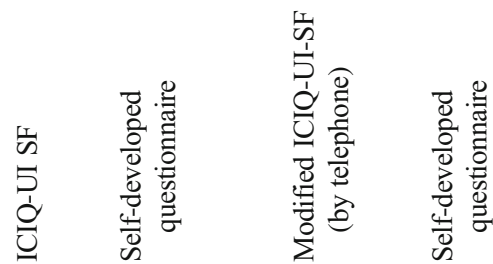
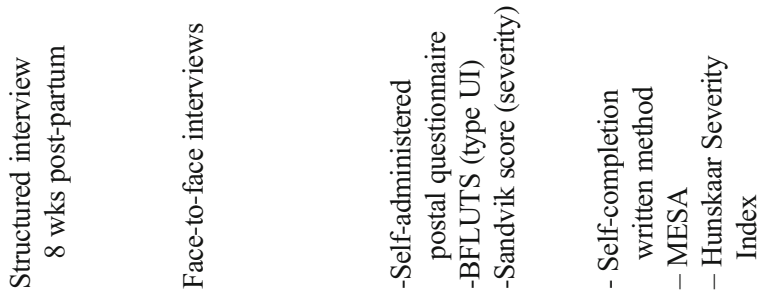

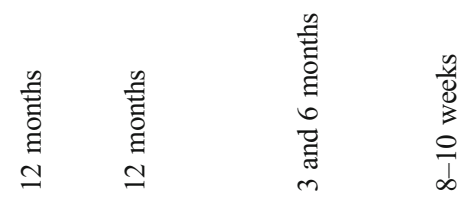

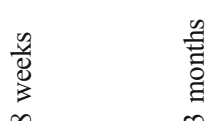

בีב

$n$
$\frac{n}{0}$
$\vdots$
0
0

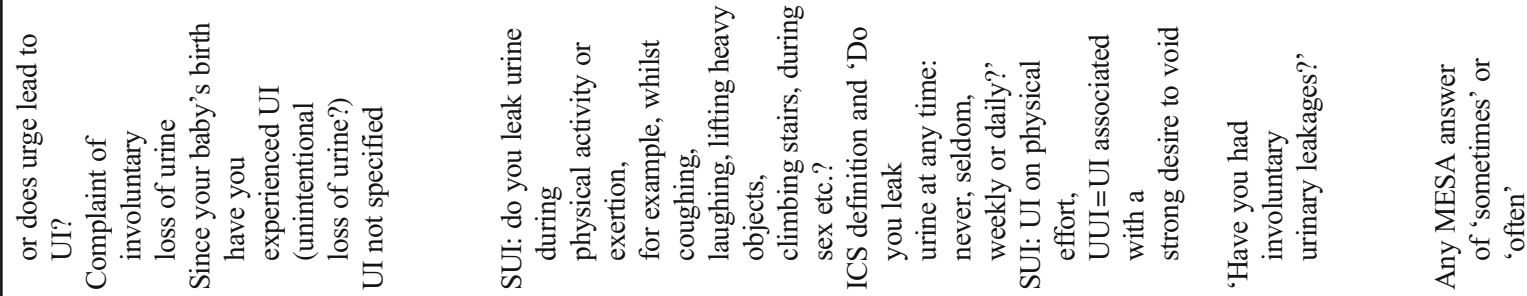

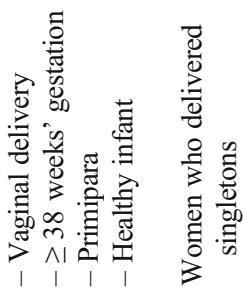

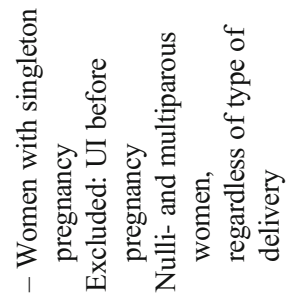

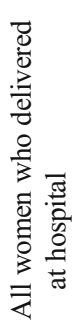

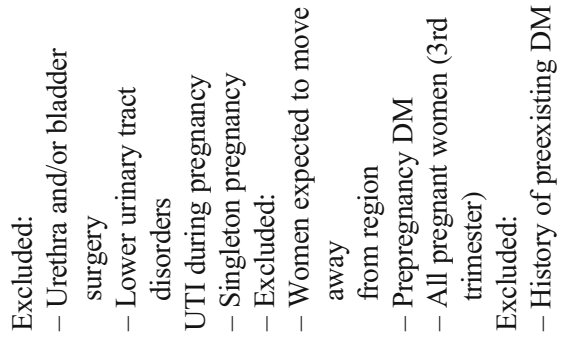

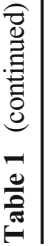

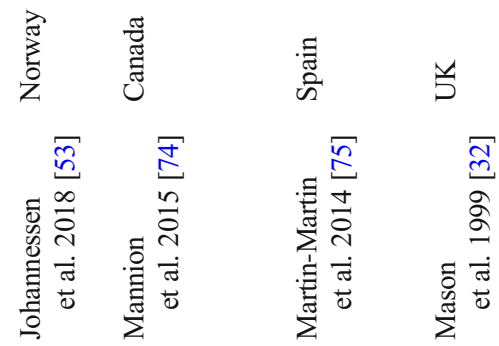

हิ

촐

导

岕

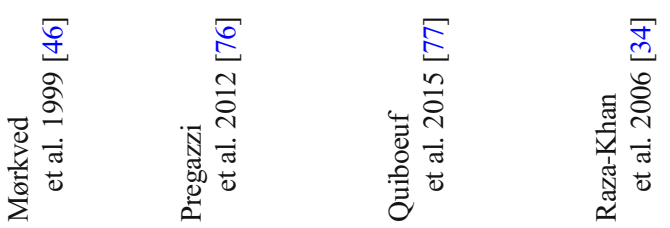




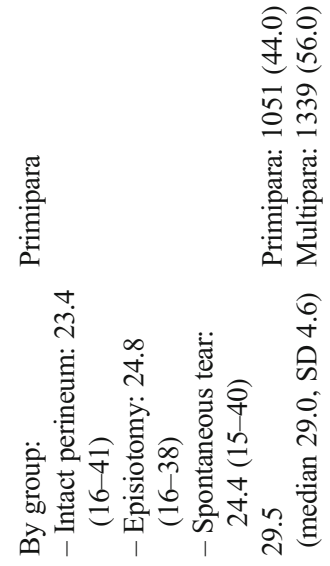

劳

营
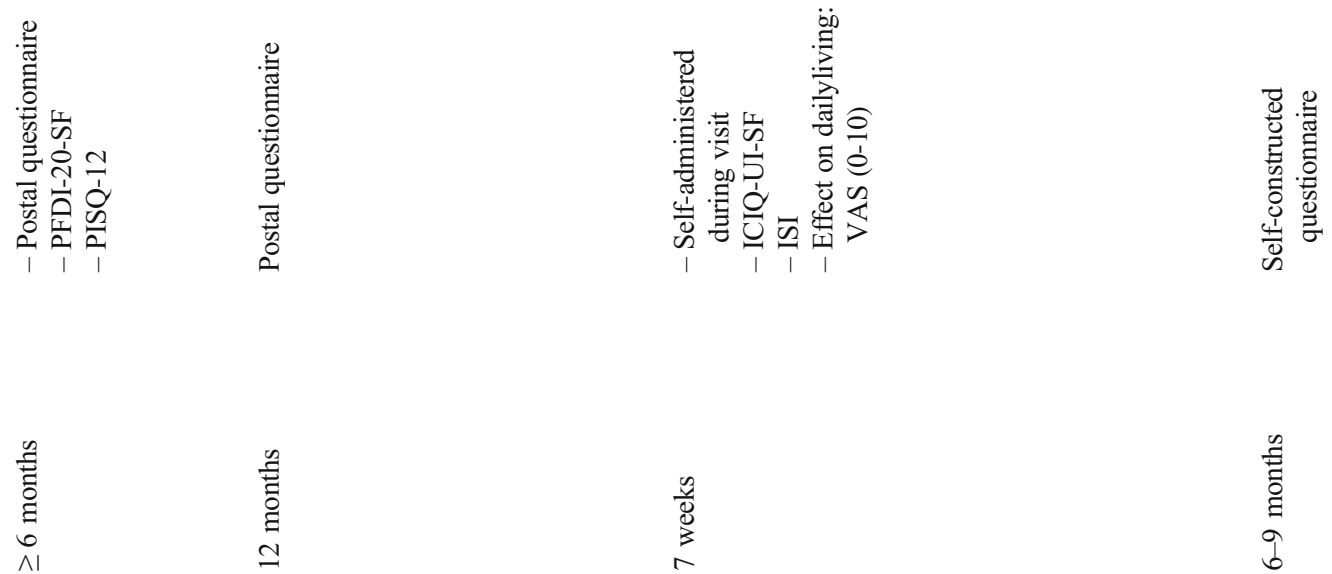

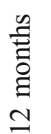

$\frac{n}{8}$

है
है
aे

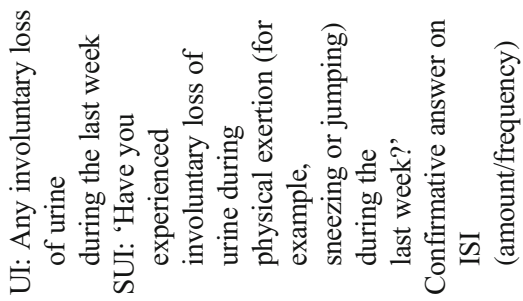

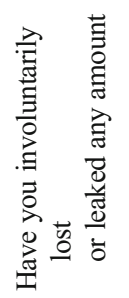
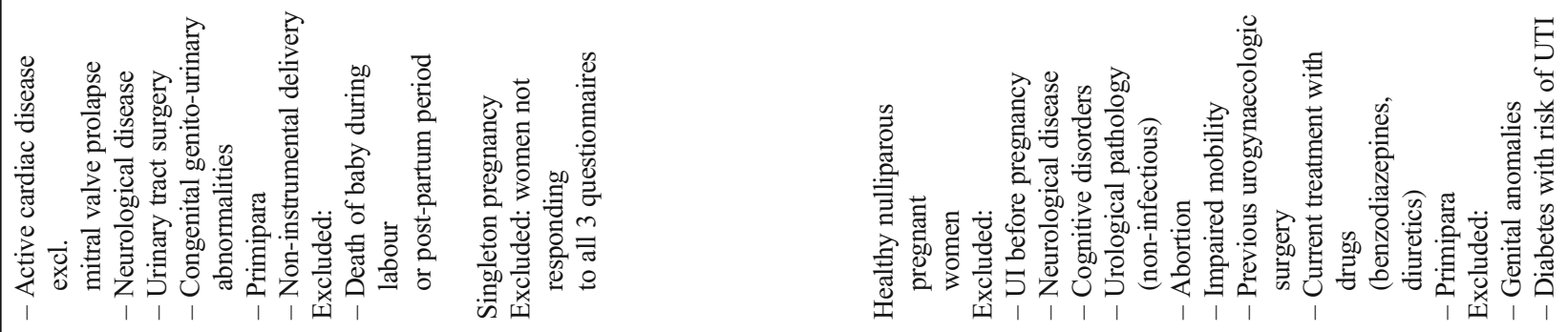

$\frac{\pi}{7}$

造

当

岕
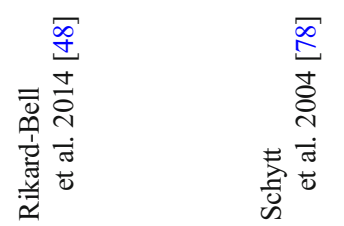

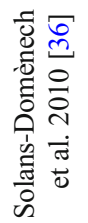

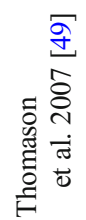



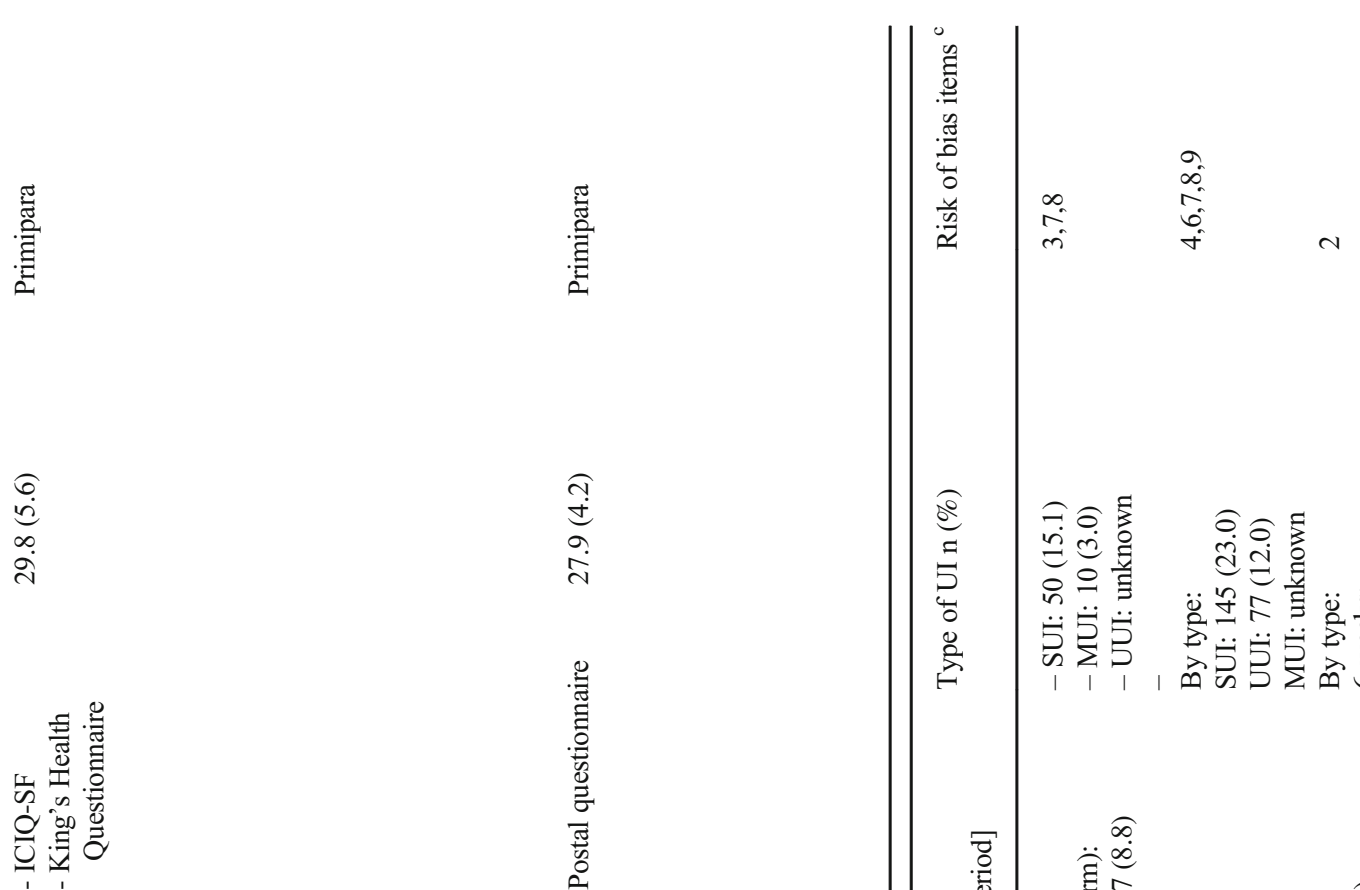

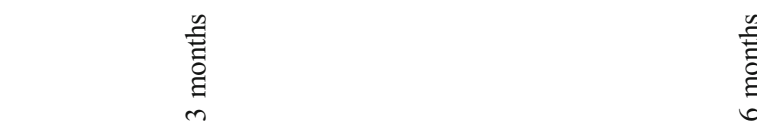

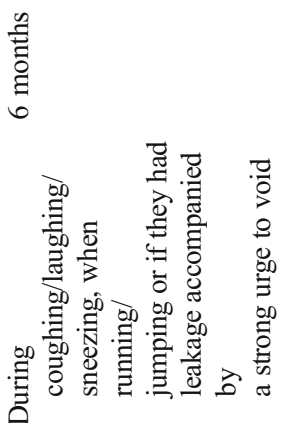

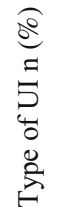

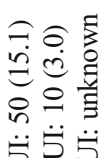

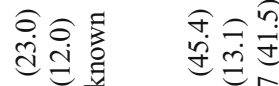

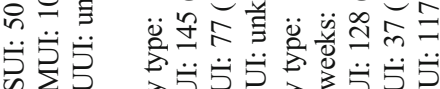

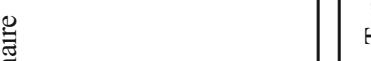

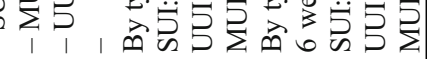

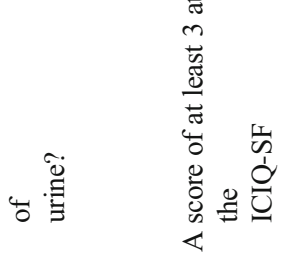

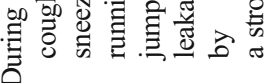

.

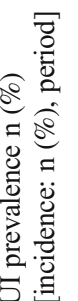

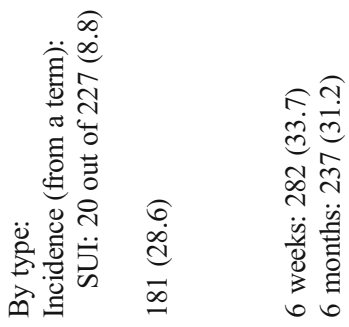

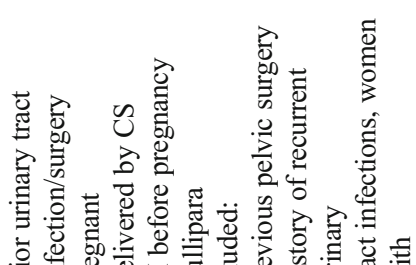

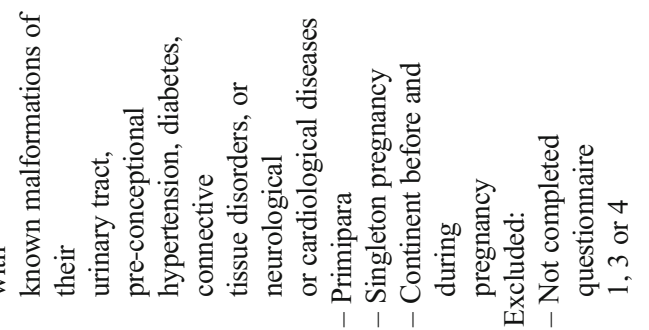

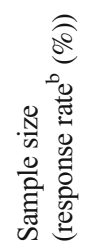

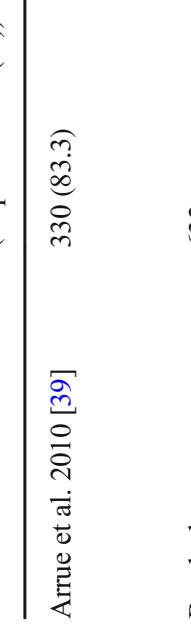

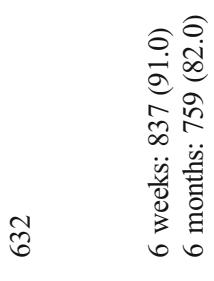

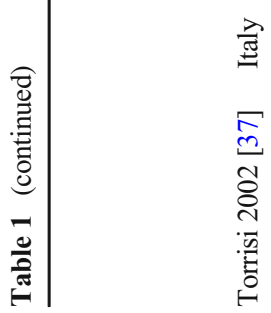

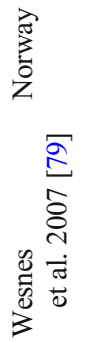

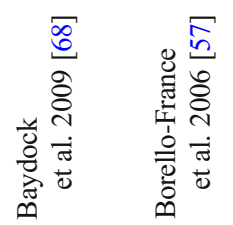




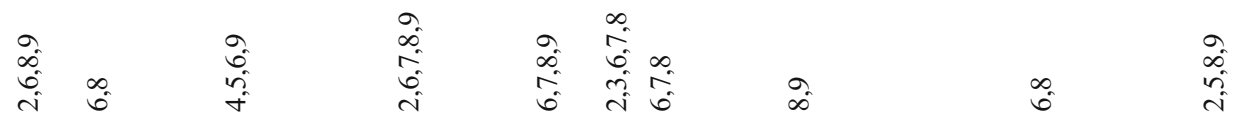

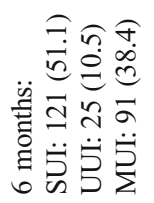
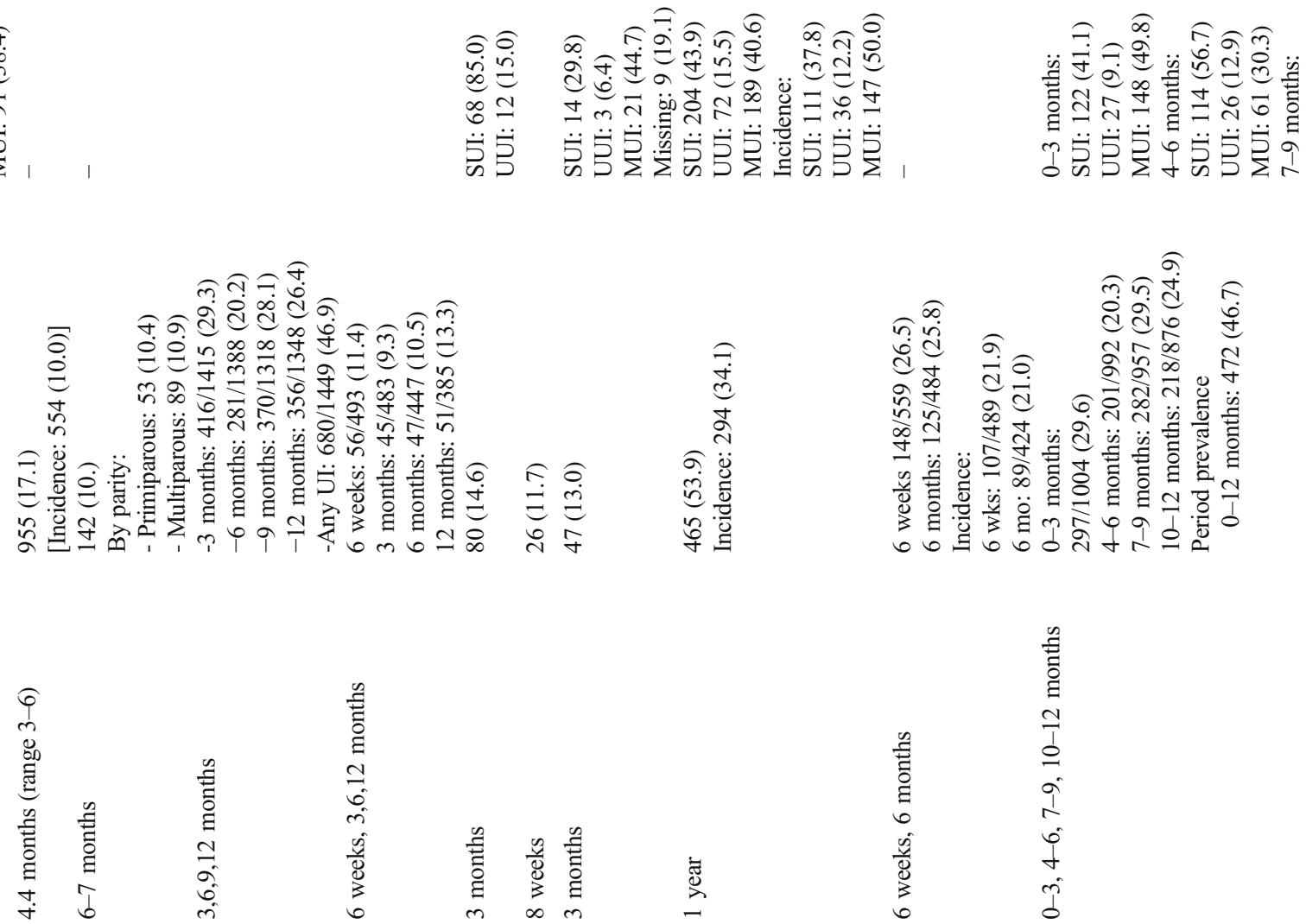

$\stackrel{\varpi}{\check{\varpi}}$

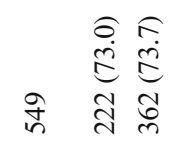

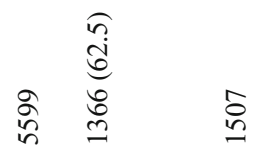

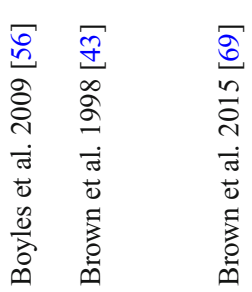

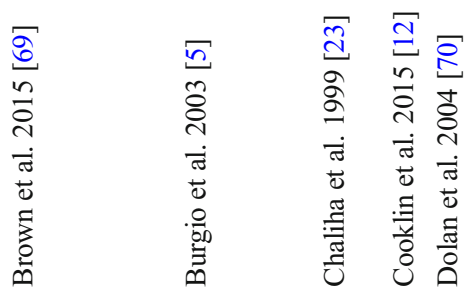

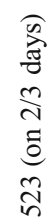

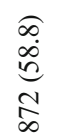

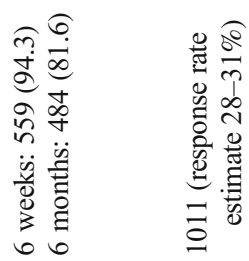

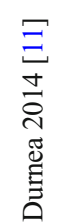

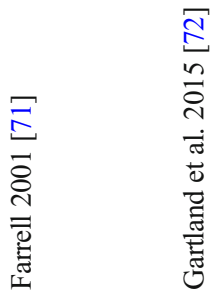




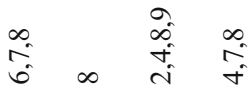
$\stackrel{\infty}{+} \stackrel{\infty}{\infty}$

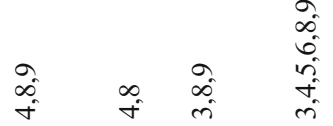

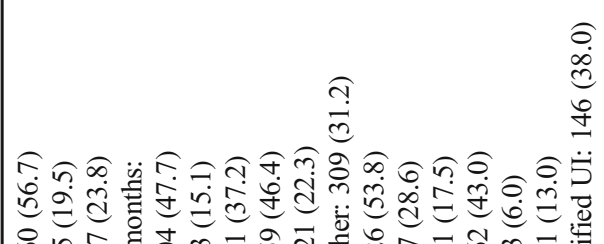

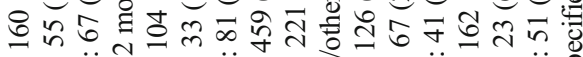

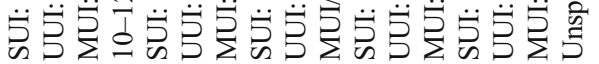

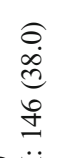

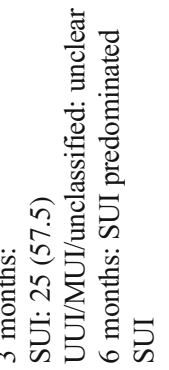

สิธ

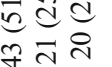

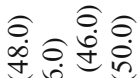
पंm

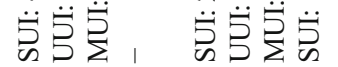

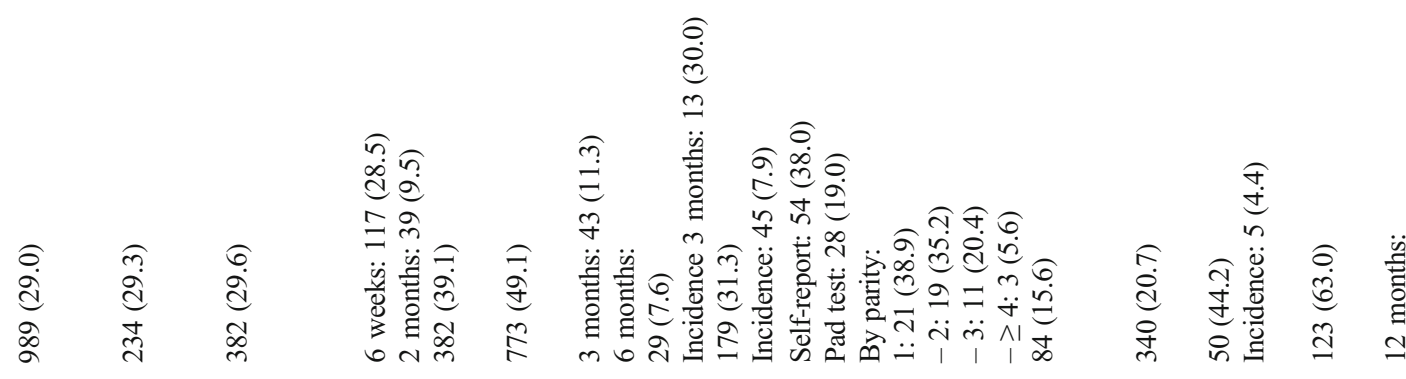

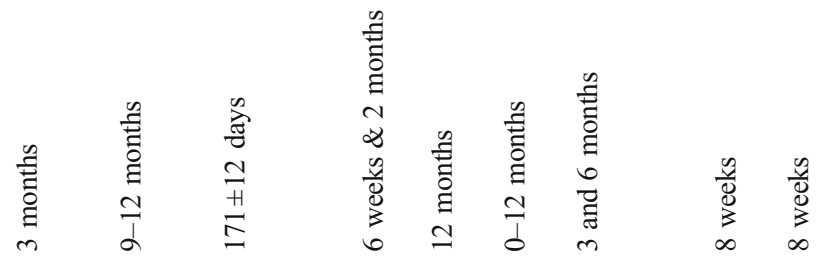
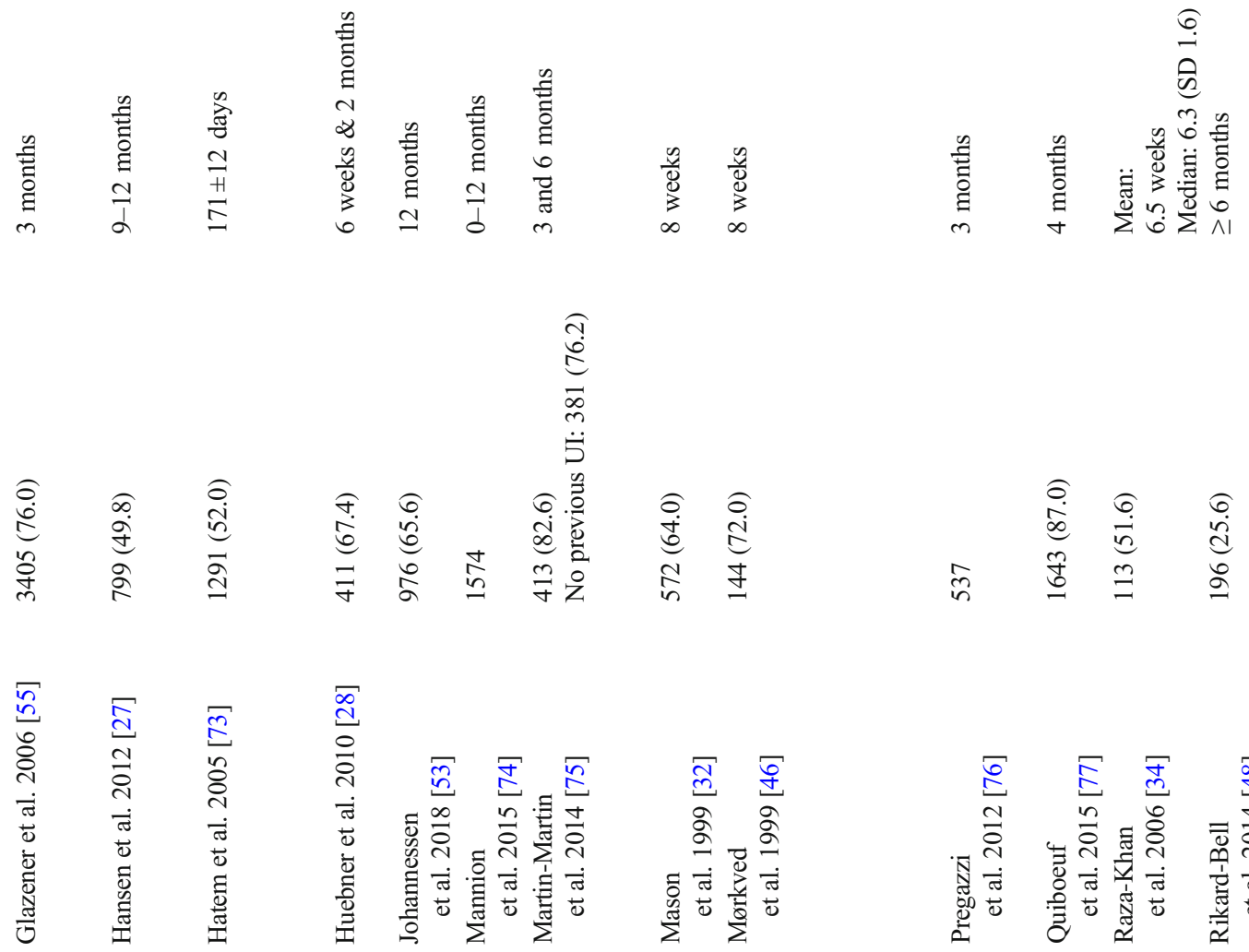

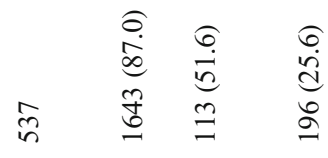

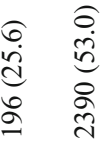

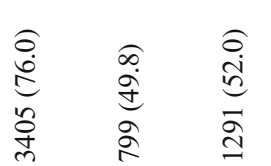

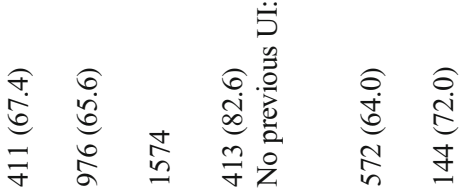
ง

$\mid$




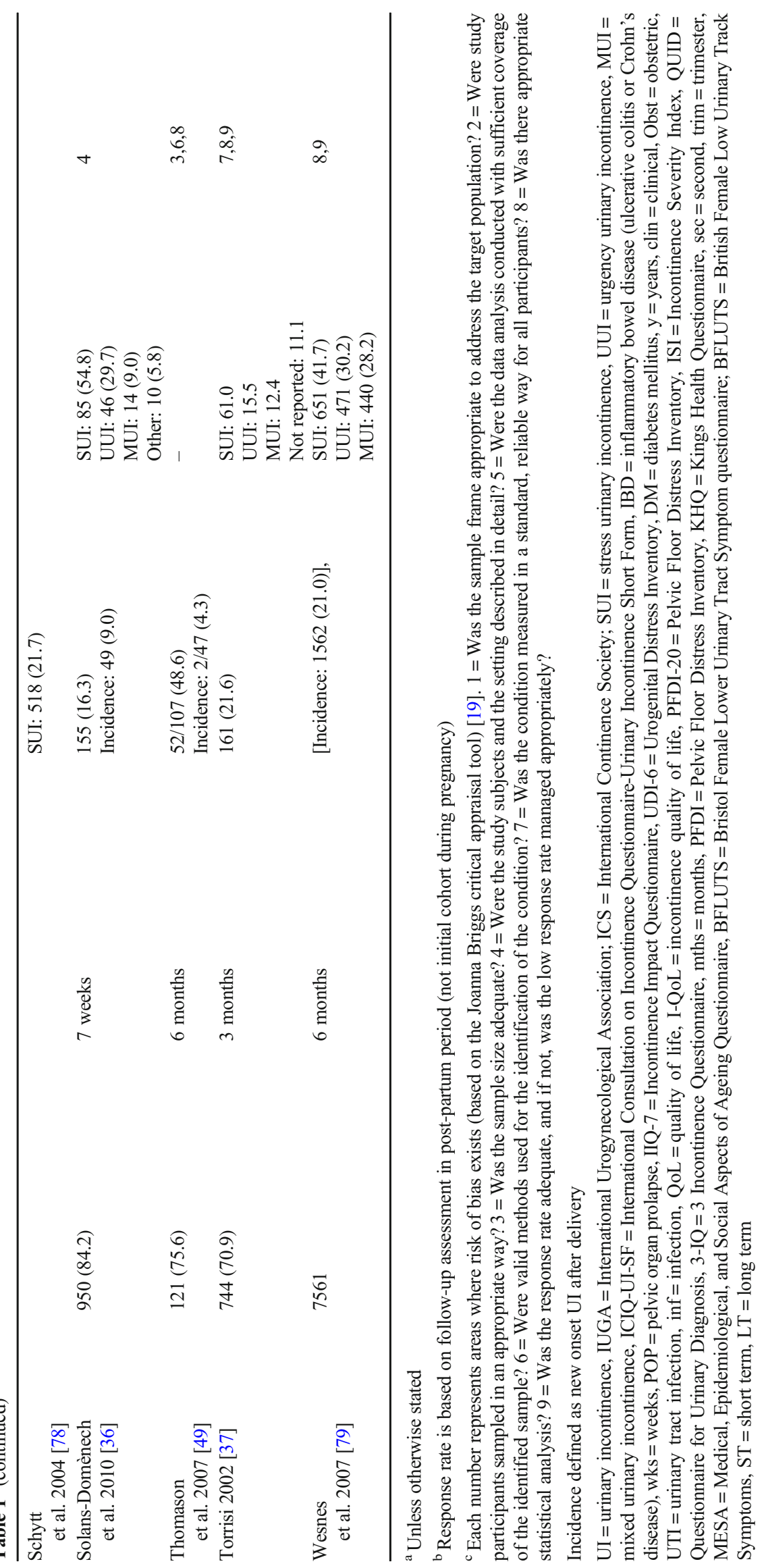


Table 2 Measurement of bother and results

\begin{tabular}{|c|c|c|c|c|c|}
\hline Measurement instrument & $\begin{array}{l}\text { Background information on } \\
\text { measurement instrument }\end{array}$ & Study & $\begin{array}{l}\text { Original measurement } \\
\text { result (mean) }\end{array}$ & Period post-partum & $\begin{array}{l}\text { (Converted) } \\
\text { measurement } \\
\text { results }(0-100)\end{array}$ \\
\hline \multirow{6}{*}{$\begin{array}{l}\text { ICIQ-UI SF } \\
(0-21)\end{array}$} & \multirow{6}{*}{$\begin{array}{l}\text { To assess symptoms of UI and impact } \\
\text { on QoL. ( } 4 \text { questions, question } 4 \text { is } \\
\text { on moment of UI and is not within } \\
\text { the calculation of the total) }\end{array}$} & [39] & 8.2 for SUI & 6 months & 39.0 \\
\hline & & & 10.0 for MUI & 6 months & 47.6 \\
\hline & & {$[27]$} & 5.9 & 1 year & 28.1 \\
\hline & & {$[53]$} & 5.1 & 1 year & 24.3 \\
\hline & & {$[37]$} & 6.0 & 3 months & 28.6 \\
\hline & & {$[50]$} & $\begin{array}{l}\text { Results reported in } \\
\text { categories. No total score }\end{array}$ & & \\
\hline \multirow{2}{*}{$\begin{array}{l}\text { ICIQ-UI SF Question } 3(\mathrm{QoL}) \\
(0-10)\end{array}$} & \multirow{2}{*}{$\begin{array}{l}\text { Question } 3 \text { of the ICIQ-UI SF is on the } \\
\text { interference in daily life of UI }\end{array}$} & {$[31]$} & 4.1 & 3 months & 41.0 \\
\hline & & & 4.5 & 6 months & 45.0 \\
\hline I-QOL & $\begin{array}{l}\text { Quality of life in persons with UI. } 3 \\
\text { subscales: } 1 \text {. Avoidance and limiting } \\
\text { behaviour, } \\
\text { 2. Psychosocial impact, } 3 \text {. Social } \\
\text { embarrassment ( } 22 \text { questions) }\end{array}$ & {$[45]$} & No exact scores reported & & \\
\hline KHQ & & {$[24]$} & No total score reported & & \\
\hline Self-constructed questionnaire & & {$[30]$} & No total score reported & & \\
\hline
\end{tabular}

ICIQ-UI-SF = International Consultation on Incontinence Questionnaire-Urinary Incontinence Short Form, QoL $=$ quality of life, I-QoL $=$ incontinence quality of life, KHQ = Kings Health Questionnaire, SUI = stress urinary incontinence, MUI = mixed urinary incontinence

Other descriptions of amount of urine lost used were: drops, small splashes and more [34, 50]. Drops were most frequently reported in $71.6 \%$ of cases [50]. The remaining study reported amount as a drop or two, pad or clothing damp, and pad or clothing soaked [5].

\section{Bother}

Nine studies reported on impact on daily life or quality of life [24, 27, 30, 31, 37, 39, 45, 50, 53], which was heterogeneously assessed. The ICIQ-UI SF total score was used most
Fig. 2 Pooled prevalence of UI post-partum

\begin{tabular}{|c|c|}
\hline \multirow{2}{*}{\multicolumn{2}{|c|}{$\begin{array}{l}\text { ES }(95 \% \mathrm{Cl}) \quad \begin{array}{l}\% \\
\text { Weight }\end{array} \\
0.29(0.25,0.32) 4.20\end{array}$}} \\
\hline & \\
\hline \multicolumn{2}{|c|}{$0.17(0.16,0.18) 4.28$} \\
\hline \multicolumn{2}{|c|}{$0.10(0.09,0.12) 4.27$} \\
\hline \multicolumn{2}{|c|}{$0.47(0.44,0.50) 4.24$} \\
\hline \multicolumn{2}{|c|}{$0.15(0.12,0.18) 4.23$} \\
\hline \multicolumn{2}{|c|}{$0.12(0.08,0.17) 4.16$} \\
\hline \multicolumn{2}{|c|}{$0.13(0.10,0.17) 4.20$} \\
\hline \multicolumn{2}{|c|}{$0.53(0.50,0.57) 4.21$} \\
\hline \multicolumn{2}{|c|}{$0.47(0.44,0.50) 4.22$} \\
\hline \multicolumn{2}{|c|}{$0.29(0.28,0.31) 4.27$} \\
\hline \multicolumn{2}{|c|}{$0.29(0.26,0.33) 4.22$} \\
\hline \multicolumn{2}{|c|}{$0.30(0.27,0.32) 4.25$} \\
\hline \multicolumn{2}{|c|}{$0.39(0.36,0.42) 4.22$} \\
\hline \multicolumn{2}{|c|}{$0.49(0.47,0.52) 4.25$} \\
\hline \multicolumn{2}{|c|}{$0.31(0.28,0.35) 4.19$} \\
\hline \multicolumn{2}{|c|}{$0.38(0.30,0.46) 3.88$} \\
\hline $0.16(0.13,0.1$ & 94.22 \\
\hline $0.21(0.19,0.2$ & 3)4.26 \\
\hline $0.44(0.35,0.5$ & 3) 3.75 \\
\hline $0.63(0.56,0.6$ & $9) 3.98$ \\
\hline $0.22(0.20,0.2$ & 3) 4.27 \\
\hline $0.16(0.14,0.1$ & 94.25 \\
\hline $0.49(0.39,0.5$ & $8) 3.72$ \\
\hline $0.22(0.19,0.2$ & $5) 4.23$ \\
\hline $0.31(0.26,0.3$ & $6) 100.00$ \\
\hline & $T$ \\
\hline & 1 \\
\hline
\end{tabular}


Fig. 3 Prevalence of UI by type and period
Prevalence by type of UI

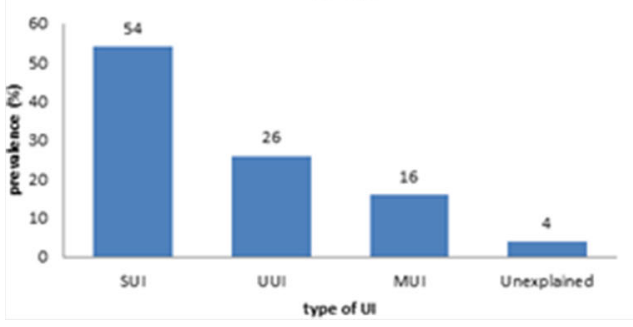

Prevalence by post-partum period

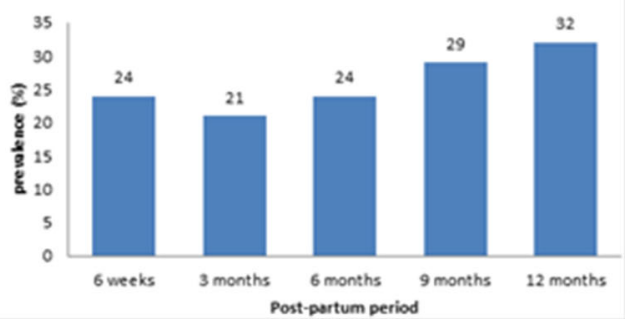

frequently $(n=5)$. Martin-Martin et al. reported the impact on daily life (0-10) based on the ICIQ-UI SF [31]. Other questionnaires used once to assess impact on daily life were: Incontinence Quality of life (I-QOL) [45], King's Health Questionnaire (KHQ) [24] and a self-constructed non-validated questionnaire [30]. The overall bother of UI post-partum, on a 0 to 100 scale, ranges between 24.3 and 47.6, consistent with mild to moderate bother. At 3 months post-partum, degree of bother ranged between 28.6 and 41.0, at 6 months post-partum between 39.0 and 45.0 and at 12 months postpartum between 24.3 and 28.1 (Table 2).

\section{Case definition}

The majority of studies ( $n=11)$ used 'any leakage' as a case definition [5, 24, 25, 27, 28, 30, 33, 49, 53, 55, 56]. Eight studies used the ICS definition, which was not mentioned as such in some cases [11, 23, 32, 35, 38, 39, 46, 47]. Six studies did not specify a case definition for UI [12, 31, 37, 43, 48, 57], five used a frequency $[22,26,34,40,50]$, and one study used the Clinical Classification of Urinary Incontinence (FPSUND) [45].

\section{Incidence}

Ten studies have examined the incidence of UI post-partum (Table 1) [11, 25, 31, 34, 36, 38, 39, 49, 54, 56]. Five studies reported incidence up to and including 3 months [25, 31, 34, $50,54]$ and six reported from 3 until 12 months $[11,25,38$, $39,49,56]$. One study reported for both periods [25]. The incidence of UI in primiparous and multiparous women up to 3 months was $9.0-21.9 \%$ and $4.4-30.0 \%$, respectively. Incidence up to 1 year was $4.3-34.1 \%$ in primiparous women.

\section{Discussion}

The aim of this systematic review and meta-analysis was to summarize the pooled prevalence and incidence of UI between 6 weeks and 12 months post-partum, to provide an overview of assessment methods for bother in relation to UI and to assess the degree of bother post-partum. The results show an overall mean prevalence rate of UI up to 1 year post-partum of $31 \%$, with a range of $10 \%$ to $63 \%$. The prevalence of $10 \%$ was reported in a study on maternal health using a generic questionnaire including only one question on UI [43] in contrast to the other studies using health problemspecific questionnaires. This might have influenced the tendency for women to report UI [58].

The prevalence numbers in the first year post-partum rise from $24 \%$ at 6 weeks to $32 \%$ at 12 months post-partum after an initial drop between 6 weeks and 3 months. A recently published systematic review and meta-analysis on the prevalence of UI during pregnancy reported a prevalence of UI of $34 \%$ in the third trimester [59]. The drop in UI prevalence early post-partum compared to the third trimester of pregnancy might be explained by the natural recovery of the levator ani muscle, which occurs mainly up to 4 to 6 months postpartum $[60,61]$. The rise in prevalence from 3 to 12 months post-partum might be due to return to daily activities, such as return to work and starting with sports, with an associated increase in physical activity level and as a consequent loading of the continence system $[62,63]$. The prevalence of UI between primi- and multiparous women was nearly equal (31\% and $30 \%$ ). This is in line with the EPINCONT study on 27,900 women, which reported that the first delivery is the largest risk factor for UI, more specifically SUI and MUI, post-partum [64].

Thom et al. published a systematic review with 33 studies on the prevalence of post-partum urinary incontinence. The overall prevalence reported by Thom et al. between 2 and 13 weeks post-partum was $33.3 \%$. As only one included study covered the period of 14 to 52 weeks post-partum, an overall prevalence number could not be calculated [13]; $33.3 \%$ is a higher prevalence than the $24 \%$ at 6 weeks and $21 \%$ at 3 months reported in this study. This might be due to the fact that Thom et al. did not report a weighted prevalence.

When interpreting the prevalence numbers at different time points post-partum, it is important to keep in mind that UI might be a dynamic phenomenon. This means that a woman's continence status can change both ways over a period of time [33].

The incidence numbers between 6 weeks up to 3 months and 3 months up to 12 months and among primi- and multiparous women varied. The low incidence number of $4.3 \%$ in the short term might be explained by the fact that this study only reported on SUI or MUI incidence [49]. Although the study of Thomason et al. claims to report the incidence of total 
UI, only women who reported UI with a positive (cough) stress test were included. Women who were able to contract their pelvic floor muscles properly and timely during an anticipated known rise in abdominal pressure might therefore be considered continent. However, these women might be incontinent during an unexpected rise in abdominal pressure. Also, the small sample ( $n=121)$ this study is based on might distort the results. If the overall incidence of the up to 3 months postpartum is compared with the up to 12 months post-partum group, the incidence numbers show a small rise in the latter, $4.3-30.0 \%$ and $4.4-34.1 \%$, respectively. The rise in incidence follows the pattern of the rise in prevalence of UI between 3 and 12 months post-partum.

Most included studies showed a moderate risk of bias, which influences the possibility to differentiate prevalence between groups regarding risk of methodological quality. The mean prevalence of UI reported by studies with low and moderate risk of bias did not differ. However, the one high risk study reported the highest prevalence of $63 \%$ [48]. Because a weighted prevalence number was calculated, this high risk study with only 196 participants and low response rate of $25.6 \%$ hardly influences the overall prevalence of UI.

The ICI recommends reporting prevalence numbers along with a measure of experienced bother [14]. Only 9 out of 31 studies (approximately 30\%) reported bother in relation to UI with a variety of measurement instruments, which shows that combined assessment is not yet common practice $[24,27,30$, $31,36,37,39,45,53]$. Eight studies used high-quality measurement instruments, most frequently the ICIQ-UI SF [27, $36,37,39,53]$. In an attempt to provide an overall assessment of degree of experienced bother in relation to UI, after studying all available materials, we decided to standardize the measurement results of different bother scales to a 0 to 100 scale. The 0 to 100 scale can be regarded as a visual analogue scale (VAS). The VAS is a valid and reproducible method to quantify the impact of UI on QoL [65], although no studies are known that report on cut-off scores for QoL specifically in post-partum women with UI. Boonstra et al. compared the VAS with a measure that assesses the impact on functioning in patients with pain and identified three classes: class 1, mild interference (score 1-34), class 2, moderate interference (score 35-64) and class 3, severe interference with daily life (score 65-100) [66]. Based on these classes, this systematic review revealed that women experience their post-partum UI as mild to moderate (range 24.3-47.6). Based on two studies, the results show a trend that bother of UI reduces at 12 months post-partum $[27,53]$. Women report for instance that UI becomes less of a problem because they get used to it and that they find practical ways to cope by using panty liners and avoiding certain activities [54].

Nevertheless, over half of the women with UI post-partum think that it will improve by itself in time and only $25 \%$ of women with post-partum UI actually seek help [67].
However, 73\% of women with UI 3 months post-partum still report UI at 6 years post-partum [6]. Reliable information on UI prevalence is thus essential to estimate health care burden, allocation of health care resources and research planning.

\section{Strengths and limitations}

The strength of this systematic review and meta-analysis is the large number of included studies, which resulted in the availability of prevalence and incidence numbers for different subpopulations (parity, post-partum period, type of UI) and for different purposes (health care providers, research planning and policy makers). This is the first review to report the prevalence and incidence over the first 12 months post-partum and bother in relation to post-partum UI.

The limitations of this study are, first, the presence of substantial clinical heterogeneity of the studies. Clinical heterogeneity may be due to differences in: case definition (any UI or different frequencies of UI in a certain period of time), population (primiparous and multiparous) or periods researched. Second, the considerable statistical heterogeneity of the studies resulted in large CIs. Third, as the Joanna Briggs critical appraisal tool does not recommend cut-off points for high, moderate or low risk of bias, we arbitrarily chose the cutoff points reported in this systematic review to explore possible differences in prevalence numbers if stratified for risk of bias. However, we did not include or exclude studies based on risk of bias.

\section{Conclusion}

After an initial drop in prevalence of UI at 3 months postpartum (21\%), at 1 year post-partum, prevalence rises again to $31 \%$. UI prevalence does not differ between primi- and multiparous women. Bother of UI is heterogeneously assessed and is reported as mild to moderate.

Supplementary Information The online version contains supplementary material available at https://doi.org/10.1007/s00192-021-04877-w.

Acknowledgements The authors thank the following persons for their support and cooperation in conducting the study: Björn Winkens, Department of Methodology and Statistics, Maastricht University, and Mrs. Julia H. Herbert for checking the English language. This study was supported by grant number 80-84300-98-72001 from The Netherlands Organization for Health Research and Development (ZonMw).

Author's contribution - HFA Moossdorff: Project development, Data collection, Data analysis, Manuscript writing.

- LCM Berghmans: Project development, Manuscript writing.

- MEA Spaanderman: Project development, Manuscript writing. 
- EMJ Bols: Project development, Data collection, Data analysis, Manuscript writing.

\section{Declarations}

\section{Conflict of interest None.}

Open Access This article is licensed under a Creative Commons Attribution 4.0 International License, which permits use, sharing, adaptation, distribution and reproduction in any medium or format, as long as you give appropriate credit to the original author(s) and the source, provide a link to the Creative Commons licence, and indicate if changes were made. The images or other third party material in this article are included in the article's Creative Commons licence, unless indicated otherwise in a credit line to the material. If material is not included in the article's Creative Commons licence and your intended use is not permitted by statutory regulation or exceeds the permitted use, you will need to obtain permission directly from the copyright holder. To view a copy of this licence, visit http://creativecommons.org/licenses/by/4.0/

\section{References}

1. Haylen BT, de Ridder D, Freeman RM, Swift SE, Berghmans B, Lee J, et al. An International Urogynecological Association (IUGA)/International Continence Society (ICS) joint report on the terminology for female pelvic floor dysfunction. Int Urogynecol J. 2010;21(1):5-26. https://doi.org/10.1007/s00192-009-0976-9.

2. Botlero R, Davis SR, Urquhart DM, Shortreed S, Bell RJ. Agespecific prevalence of, and factors associated with, different types of urinary incontinence in community-dwelling Australian women assessed with a validated questionnaire. Maturitas. 2009;62(2): 134-9. https://doi.org/10.1016/j.maturitas.2008.12.017.

3. Viktrup L, Rortveit G, Lose G. Risk of stress urinary incontinence twelve years after the first pregnancy and delivery. Obstet Gynecol. 2006;108(2):248-54. https://doi.org/10.1097/01.AOG. $0000226860.01127 .0 \mathrm{e}$.

4. Linde JM, Nijman RJM, Trzpis M, Broens PMA. Urinary incontinence in the Netherlands: prevalence and associated risk factors in adults. Neurourol Urodyn. 2017;36(6):1519-28. https://doi.org/10. 1002/nau.23121.

5. Burgio KL, Zyczynski H, Locher JL, Richter HE, Redden DT, Wright KC. Urinary incontinence in the 12-month postpartum period. Obstet Gynecol. 2003;102(6):1291-8. https://doi.org/10. 1016/j.obstetgynecol.2003.09.013.

6. MacArthur C, Wilson D, Herbison P, Lancashire RJ, Hagen S, Toozs-Hobson $\mathrm{P}$, et al. Urinary incontinence persisting after childbirth: extent, delivery history, and effects in a 12-year longitudinal cohort study. Bjog. 2016;123(6):1022-9. https://doi.org/10.1111/ 1471-0528.13395.

7. Ebbesen MH, Hunskaar S, Rortveit G, Hannestad YS. Prevalence, incidence and remission of urinary incontinence in women: longitudinal data from the Norwegian HUNT study (EPINCONT). BMC Urol. 2013;13:27. https://doi.org/10.1186/1471-2490-13-27.

8. Handa VL, Zyczynski HM, Burgio KL, Fitzgerald MP, BorelloFrance D, Janz NK, et al. The impact of fecal and urinary incontinence on quality of life 6 months after childbirth. Am J Obstet Gynecol. 2007;197(6):636.e631-6.

9. Coyne KS, Wein A, Nicholson S, Kvasz M, Chen CI, Milsom I. Economic burden of urgency urinary incontinence in the United States: a systematic review. J Manag Care Pharm. 2014;20(2): 130-40. https://doi.org/10.18553/jmcp.2014.20.2.130.
10. Mendes A, Hoga L, Gonçalves B, Silva P, Pereira P. Adult women's experiences of urinary incontinence: a systematic review of qualitative evidence. JBI Database System Rev Implement Rep. 2017;15(5):1350-408. https://doi.org/10.11124/jbisrir-2017003389.

11. Durnea CM, Khashan AS, Kenny LC, Tabirca SS, O'Reilly BA. The role of prepregnancy pelvic floor dysfunction in postnatal pelvic morbidity in primiparous women. Int Urogynecol J. 2014;25(10):1363-74. https://doi.org/10.1007/s00192-014-23812.

12. Cooklin AR, Amir LH, Jarman J, Cullinane M, Donath SM. Maternal physical health symptoms in the first 8 weeks postpartum among primiparous Australian women. Birth. 2015;42(3):254-60. https://doi.org/10.1111/birt.12168.

13. Thom DH, Rortveit G. Prevalence of postpartum urinary incontinence: a systematic review. Acta Obstet Gynecol Scand. 2010;89(12):1511-22. https://doi.org/10.3109/00016349.2010. 526188 .

14. Abrams A, Cardozo L, Wagg A, Wein A, editors. Incontinence 6th edition. Bristol: ICI-ICS. International Continence Society; 2017.

15. Hägglund D, Walker-Engström ML, Larsson G, Leppert J. Quality of life and seeking help in women with urinary incontinence. Acta Obstet Gynecol Scand. 2001;80(11):1051-5.

16. Stroup DF, Berlin JA, Morton SC, Olkin I, Williamson GD, Rennie D, et al. Meta-analysis of observational studies in epidemiology: a proposal for reporting. Meta-analysis of observational studies in epidemiology (MOOSE) group. JAMA. 2000;283(15):2008-12. https://doi.org/10.1001/jama.283.15.2008.

17. Portney LG, Watkins MP (2009) Foundations of Clinical Research. 3 edn. Pearson Prentice Hall, Upper Saddle River, USA

18. (CPH) CPH (2020) Data Extraction and Assessment Template. https://ph.cochrane.org/review-authors.

19. Munn Z, Moola S, Riitano D, Lisy K. The development of a critical appraisal tool for use in systematic reviews addressing questions of prevalence. Int J Health Policy Manag. 2014;3(3):123-8. https:// doi.org/10.15171/ijhpm.2014.71.

20. Munn Z, Moola S, Lisy K, Riitano D, Tufanaru C. Methodological guidance for systematic reviews of observational epidemiological studies reporting prevalence and cumulative incidence data. Intl $\mathrm{J}$ Evidence-Based Healthcare. 2015;13(3):147-53. https://doi.org/10. 1097/xeb.0000000000000054.

21. Cochrane Handbook for Systematic Reviews of Interventions version 6.0 (2019).

22. Brown S, Gartland D, Perlen S, McDonald E, MacArthur C. Consultation about urinary and faecal incontinence in the year after childbirth: a cohort study. BJOG: An International Journal of Obstetrics and Gynaecology. 2015;122(7):954-62. https://doi.org/ 10.1111/1471-0528.12963.

23. Chaliha C, Kalia V, Stanton S, Monga A, Sultan A. Antenatal prediction of postpartum urinary and fecal incontinence. Obstet Gynecol. 1999;94(5):689-94.

24. Dolan LM, Walsh D, Hamilton S, Marshall K, Thompson K, Ashe RG. A study of quality of life in primigravidae with urinary incontinence. Intl Urogynecol J. 2004;15(3):160-4. https://doi.org/10. 1007/s00192-004-1128-x.

25. Farrell SA, Allen VM, Baskett TF. Parturition and urinary incontinence in primiparas. Obstet Gynecol. 2001;97(3):350-6. https:// doi.org/10.1016/S0029-7844\%2800\%2901164-9.

26. Gartland D, MacArthur C, Woolhouse H, McDonald E, Brown SJ. Frequency, severity and risk factors for urinary and faecal incontinence at 4 years postpartum: a prospective cohort. BJOG: Intl J Obstet Gynaecol. 2016;123(7):1203-11. https://doi.org/10.1111/ 1471-0528.13522.

27. Hansen BB, Svare J, Viktrup L, Jorgensen T, Lose G. Urinary incontinence during pregnancy and 1 year after delivery in primiparous women compared with a control group of nulliparous 
women. Neurourol Urodyn. 2012;31(4):475-80. https://doi.org/10. 1002/nau.21221.

28. Huebner M, Antolic A, Tunn R. The impact of pregnancy and vaginal delivery on urinary incontinence. Int $\mathrm{J}$ Gynecol Obstet. 2010;110(3):249-51. https://doi.org/10.1016/j.ijgo.2010.04.019.

29. Johannessen HH, Wibe A, Stordahl A, Morkved S. Prevalence of anal and urinary incontinence in primiparas and impact on health related quality of life. Neurourol Urodynam. 2012;31(6):1089-91. https://doi.org/10.1002/nau.22287.

30. Mannion CA, Vinturache AE, McDonald SW, Tough SC (2015) The influence of back pain and urinary incontinence on daily tasks of mothers at 12 months postpartum. PLoS ONE 10 (6) (no pagination) (e0129615). doi:https://doi.org/10.1371/journal.pone. 0129615

31. Martin-Martin S, Pascual-Fernandez A, Alvarez-Colomo C, CalvoGonzalez R, Munoz-Moreno M, Cortinas-Gonzalez JR. Urinary incontinence during pregnancy and postpartum. Associated risk factors and influence of pelvic floor exercises. Archivos espanoles de urologia. 2014;67(4):323-30.

32. Mason L, Glenn S, Walton I, Appleton C. The prevalence of stress incontinence during pregnancy and following delivery. Midwifery. 1999;15(2):120-8. https://doi.org/10.1016/s0266-6138(99)900086.

33. Quiboeuf E, Saurel-Cubizolles MJ, Fritel X. Trends in urinary incontinence in women between 4 and 24 months postpartum in the EDEN cohort. BJOG: An International Journal of Obstetrics and Gynaecology. 2016;123(7):1222-8. https://doi.org/10.1111/14710528.13545 .

34. Raza-Khan F, Graziano S, Kenton K, Shott S, Brubaker L. Peripartum urinary incontinence in a racially diverse obstetrical population. Int Urogynecol J Pelvic Floor Dysfunct. 2006;17(5): 525-30. https://doi.org/10.1007/s00192-005-0061-y.

35. Schytt E, Lindmark G, Waldenstrom U. Symptoms of stress incontinence 1 year after childbirth: prevalence and predictors in a national Swedish sample. Acta Obstet Gynecol Scand. 2004;83(10): 928-36. https://doi.org/10.1111/j.0001-6349.2004.00431.x.

36. Solans-Domènech M, Sánchez E, Espuña-Pons M. Urinary and anal incontinence during pregnancy and postpartum: incidence, severity, and risk factors. Obstet Gynecol. 2010;115(3):618-28. https://doi.org/10.1097/AOG.0b013e3181d04dff.

37. Torrisi G, Minini G, Bernasconi F, Perrone A, Trezza G, Guardabasso V, et al. A prospective study of pelvic floor dysfunctions related to delivery. Eur J Obstet Gynecol Reprod Biol. 2012;160(1):110-5. https://doi.org/10.1016/j.ejogrb.2011.10.010.

38. Wesnes SL, Hunskaar S, Bo K, Rortveit G. Urinary incontinence and weight change during pregnancy and postpartum: a cohort study. Am J Epidemiol. 2010;172(9):1034-44. https://doi.org/10. 1093/aje/kwq240.

39. Arrue M, Ibañez L, Paredes J, Murgiondo A, Belar M, Sarasqueta $\mathrm{C}$, et al. Stress urinary incontinence six months after first vaginal delivery. Eur J Obstet Gynecol Reprod Biol. 2010;150(2):210-4. https://doi.org/10.1016/j.ejogrb.2010.02.039.

40. Baydock SA, Flood C, Schulz JA, MacDonald D, Esau D, Jones S, et al. Prevalence and risk factors for urinary and fecal incontinence four months after vaginal delivery. J Obstet Gynaecol Canada. 2009;31(1):36-41. https://doi.org/10.1016/S1701-2163\%2816\% 2934051-8.

41. Borello-France D, Burgio KL, Richter HE, Zyczynski H, FitzGerald MP, Whitehead W, et al. Fecal and urinary incontinence in primiparous women. Obstet Gynecol. 2006;108(4):863-72.

42. Boyles SH, Li H, Mori T, Osterweil P, Guise JM, Boyles SH, et al. Effect of mode of delivery on the incidence of urinary incontinence in primiparous women. Obstet Gynecol. 2009;113(1):134-41. https://doi.org/10.1097/AOG.0b013e318191bb37.

43. Brown S, Lumley J. Maternal health after childbirth: results of an Australian population based survey. Br J Obstet Gynaecol.
1998;105(2):156-61. https://doi.org/10.1111/j.1471-0528.1998. tb10045.x.

44. Glazener CMA, Herbison GP, MacArthur C, Lancashire R, McGee MA, Grant AM, et al. New postnatal urinary incontinence: obstetric and other risk factors in primiparae. BJOG: Intl J Obstet Gynaecol. 2006;113(2):208-17.

45. Hatem M, Fraser W, Lepire E. Postpartum urinary and anal incontinence: a population-based study of quality of life of Primiparous women in Quebec. J Obstet GynaecolCanada. 2005;27(7):682-8. https://doi.org/10.1016/S1701-2163\%2816\%2930546-1.

46. Morkved S, Bo K. Prevalence of urinary incontinence during pregnancy and postpartum. Intl Urogynecol J. 1999;10(6):394-8. https://doi.org/10.1007/s001920050067.

47. Pregazzi R, Sartore A, Troiano L, Grimaldi E, Bortoli P, Siracusano $\mathrm{S}$, et al. Postpartum urinary symptoms: prevalence and risk factors. Eur J Obstet Gynecol Reproduct Biol. 2002;103(2):179-82. https:// doi.org/10.1016/S0301-2115\%2802\%2900045-3.

48. Rikard-Bell J, Iyer J, Rane A. Perineal outcome and the risk of pelvic floor dysfunction: a cohort study of primiparous women. Australian and New Zealand J Obstet Gynaecol. 2014;54(4):3716. https://doi.org/10.1111/ajo.12222.

49. Thomason AD, Miller JM, Delancey JO. Urinary incontinence symptoms during and after pregnancy in continent and incontinent primiparas. Int Urogynecol J Pelvic Floor Dysfunct. 2007;18(2): 147-51. https://doi.org/10.1007/s00192-006-0124-8.

50. Solans-Domenech M, Sanchez E, Espuna-Pons M (2010) URinary and anal incontinence during pregnancy and postpartum: incidence, severity, and risk factors. Obstet Gynecol 115 (3):618-628. doi: https://doi.org/10.1097/AOG.0b013e3181d04dff

51. Hojberg KE, Salvig JD, Winslow NA, Lose G, Secher NJ. Urinary incontinence: prevalence and risk factors at 16 weeks of gestation. Br J Obstet Gynaecol. 1999;106(8):842-50. https://doi.org/10. 1111/j.1471-0528.1999.tb08407.x.

52. Nigam A, Ahmad A, Gaur D, Elahi AA, Batra S. Prevalence and risk factors for urinary incontinence in pregnant women during late third trimester. Intl J Reproduct , Contracep, Obstet Gynecol. 2016;5(7):2187-91. https://doi.org/10.18203/2320-1770. ijcog20162090.

53. Johannessen HH, Stafne SN, Falk RS, Stordahl A, Wibe A, Mørkved S. Prevalence and predictors of double incontinence 1 year after first delivery. Int Urogynecol J. 2018;29(10):1529-35. https://doi.org/10.1007/s00192-018-3577-7.

54. Mason L, Glenn S, Walton I, Appleton C. The experience of stress incontinence after childbirth. Birth: Issues in Perinatal Care. 1999;26(3):164-71.

55. Glazener CM, Herbison GP, MacArthur C, Lancashire R, McGee MA, Grant AM, et al. New postnatal urinary incontinence: obstetric and other risk factors in primiparae. Bjog. 2006;113(2):208-17. https://doi.org/10.1111/j.1471-0528.2005.00840.x.

56. Boyles SH, Li H, Mori T, Osterweil P, Guise JM. Effect of mode of delivery on the incidence of urinary incontinence in primiparous women. Obstet Gynecol. 2009;113(1):134-41. https://doi.org/10. 1097/AOG.0b013e318191bb37.

57. Borello-France D, Burgio KL, Richter HE, Zyczynski H, FitzGerald MP, Whitehead W, et al. Fecal and urinary incontinence in primiparous women. Obstet Gynecol. 2006;108(4):863-72. https://doi.org/10.1097/01.AOG.0000232504.32589.3b.

58. Streiner D, Norman G (1989) Health measurement scales. Fourth Edition edn. Oxford University Press, Oxford, UK

59. Moossdorff-Steinhauser HFA, Berghmans BCM, Spaanderman MEA, Bols EMJ. Prevalence, incidence and bothersomeness of urinary incontinence in pregnancy: a systematic review and metaanalysis. Int Urogynecol J. 2021. https://doi.org/10.1007/s00192020-04636-3.

60. Stær-Jensen J, Siafarikas F, Hilde G, Benth J, Bø K, Engh ME. Postpartum recovery of levator hiatus and bladder neck mobility 
in relation to pregnancy. Obstet Gynecol. 2015;125(3):531-9. https://doi.org/10.1097/aog.0000000000000645.

61. Shek KL, Chantarasorn V, Langer S, Dietz HP. Does levator trauma 'heal'? Ultrasound Obstet Gynecol Official J Intl Soc Ultrasound Obstet Gynecol. 2012;40(5):570-5. https://doi.org/10.1002/uog. 11203.

62. Hesketh KR, Evenson KR, Stroo M, Clancy SM, Østbye T, Benjamin-Neelon SE. Physical activity and sedentary behavior during pregnancy and postpartum, measured using hip and wrist-worn accelerometers. Prevent Med Rep. 2018;10:337-45. https://doi.org/ 10.1016/j.pmedr.2018.04.012.

63. Evenson KR, Herring AH, Wen F. Self-reported and objectively measured physical activity among a cohort of postpartum women: the PIN postpartum study. J Phys Activity Health. 2012;9(1):5-20. https://doi.org/10.1123/jpah.9.1.5.

64. Rortveit G, Hannestad YS, Daltveit AK, Hunskaar S. Age- and type-dependent effects of parity on urinary incontinence: the Norwegian EPINCONT study. Obstet Gynecol. 2001;98(6):100410. https://doi.org/10.1016/s0029-7844(01)01566-6.

65. Stach-Lempinen B, Kujansuu E, Laippala P, Metsänoja R. Visual analogue scale, urinary incontinence severity score and $15 \mathrm{D}$ psychometric testing of three different health-related quality-oflife instruments for urinary incontinent women. Scandinavian J Urol Nephrol. 2001;35(6):476-83. https://doi.org/10.1080/ 003655901753367587.

66. Boonstra AM, Schiphorst Preuper HR, Balk GA, Stewart RE. Cutoff points for mild, moderate, and severe pain on the visual analogue scale for pain in patients with chronic musculoskeletal pain. Pain. 2014;155(12):2545-50. https://doi.org/10.1016/j.pain.2014. 09.014 .

67. Moossdorff-Steinhauser HFA, Berghmans BCM, Spaanderman MEA, Bols EMJ. Urinary incontinence 6 weeks to 1 year postpartum: prevalence, experience of bother, beliefs, and helpseeking behavior. Int Urogynecol J. 2021. https://doi.org/10.1007/ s00192-020-04644-3.

68. Baydock SA, Flood C, Schulz JA, MacDonald D, Esau D, Jones S, et al. Prevalence and risk factors for urinary and fecal incontinence four months after vaginal delivery. J Obstet Gynaecol Can. 2009;31(1):36-41. https://doi.org/10.1016/s1701-2163(16)340518.

69. Brown S, Gartland D, Perlen S, McDonald E, MacArthur C. Consultation about urinary and faecal incontinence in the year after childbirth: a cohort study. Bjog. 2015;122(7):954-62. https://doi. org/10.1111/1471-0528.12963.
70. Dolan LM, Walsh D, Hamilton S, Marshall K, Thompson K, Ashe RG. A study of quality of life in primigravidae with urinary incontinence. Int Urogynecol J Pelvic Floor Dysfunct. 2004;15(3):160-4. https://doi.org/10.1007/s00192-004-1128-x.

71. Farrell SA, Allen VM, Baskett TF. Parturition and urinary incontinence in primiparas. Obstet Gynecol. 2001;97(3):350-6. https:// doi.org/10.1016/s0029-7844(00)01164-9.

72. Gartland D, MacArthur C, Woolhouse H, McDonald E, Brown SJ. Frequency, severity and risk factors for urinary and faecal incontinence at 4 years postpartum: a prospective cohort. Bjog. 2016;123(7):1203-11. https://doi.org/10.1111/1471-0528.13522.

73. Hatem M, Fraser W, Lepire E. Postpartum urinary and anal incontinence: a population-based study of quality of life of primiparous women in Quebec. J Obstet Gynaecol Can. 2005;27(7):682-8. https://doi.org/10.1016/s1701-2163(16)30546-1.

74. Mannion CA, Vinturache AE, McDonald SW, Tough SC. The influence of Back pain and urinary incontinence on daily tasks of mothers at 12 months postpartum. PLoS One. 2015;10(6): e0129615. https://doi.org/10.1371/journal.pone.0129615.

75. Martin-Martin S, Pascual-Fernandez A, Alvarez-Colomo C, CalvoGonzalez R, Muñoz-Moreno M, Cortiñas-Gonzalez JR. Urinary incontinence during pregnancy and postpartum. Associated risk factors and influence of pelvic floor exercises. Arch Esp Urol. 2014;67(4):323-30.

76. Pregazzi R, Sartore A, Troiano L, Grimaldi E, Bortoli P, Siracusano $\mathrm{S}$, et al. Postpartum urinary symptoms: prevalence and risk factors. Eur J Obstet Gynecol Reprod Biol. 2002;103(2):179-82. https:// doi.org/10.1016/s0301-2115(02)00045-3.

77. Quiboeuf E, Saurel-Cubizolles MJ, Fritel X. Trends in urinary incontinence in women between 4 and 24 months postpartum in the EDEN cohort. Bjog. 2016;123(7):1222-8. https://doi.org/10.1111/ 1471-0528.13545.

78. Schytt E, Lindmark G, Waldenström U. Symptoms of stress incontinence 1 year after childbirth: prevalence and predictors in a national Swedish sample. Acta Obstet Gynecol Scand. 2004;83(10): 928-36. https://doi.org/10.1111/j.0001-6349.2004.00431.x.

79. Wesnes SL, Hannestad Y, Rortveit G. Delivery parameters, neonatal parameters and incidence of urinary incontinence six months postpartum: a cohort study. Acta Obstet Gynecol Scand. 2017;96(10):1214-22. https://doi.org/10.1111/aogs.13183.

Publisher's note Springer Nature remains neutral with regard to jurisdictional claims in published maps and institutional affiliations. 